\title{
Shadowing is Generic on Various One-Dimensional Continua with a Special Geometric Structure
}

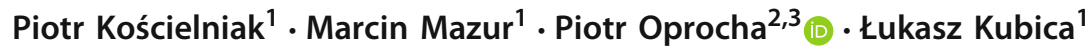

Received: 16 April 2018 / Published online: 27 September 2019

(c) The Author(s) 2019

\begin{abstract}
In the paper we use a special geometric structure of selected one-dimensional continua to prove that some stronger versions of the shadowing property are generic (or at least dense) for continuous maps acting on these spaces. Specifically, we prove that (i) the periodic $\mathscr{T}_{S}$-bi-shadowing property, where $\mathscr{T}_{S}$ means some class of continuous methods, is generic as well as the s-limit shadowing property is dense in the space of all continuous maps (and all continuous surjective maps) of any topological graph; (ii) the $\mathscr{T}_{S}$-bi-shadowing property is generic as well as the s-limit shadowing property is dense in the space of all continuous maps of any dendrite; (iii) the $\mathscr{T}_{S}$-bi-shadowing property is generic in the space of all continuous maps of chainable continuum that can by approximated by arcs from the inside. The results of the paper extend ones obtained over the last few decades by various authors (see, e.g., Kościelniak in J Math Anal Appl 310:188-196, 2005; Kościelniak and Mazur in J Differ Equ Appl 16:667674, 2010; Kościelniak et al. in Discret Contin Dyn Syst 34:3591-3609, 2014; Mazur and Oprocha in J Math Anal Appl 408:465-475, 2013; Mizera in Generic Properties of One-Dimensional Dynamical Systems, Ergodic Theory and Related Topics, III, Springer, Berlin, 1992; Odani in Proc Am Math Soc 110:281-284, 1990; Pilyugin and Plamenevskaya in Topol Appl 97:253-266, 1999; and Yano in J Fac Sci Univ Tokyo Sect IA Math 34:51-55, 1987) for both homeomorphisms and continuous maps of compact manifolds, including (in particular) an interval and a circle, which are the simplest examples of one-dimensional continua. Moreover, from a technical point of view our considerations are a continuation of those carried out in the earlier work by Mazur and Oprocha in J. Math. Anal. Appl. 408:465-475, 2013.
\end{abstract}

Keywords Dynamical system · Continuous map · Pseudo-orbit · S-limit shadowing • Periodic shadowing $\cdot$ Inverse shadowing $\cdot \mathscr{C}^{0}$-genericity $\cdot$ Graph $\cdot$ Dendrite

Chainable continuum

Mathematics Subject Classification $37 \mathrm{C} 50 \cdot 37 \mathrm{C} 20 \cdot 37 \mathrm{~B} 45 \cdot 54 \mathrm{H} 20 \cdot 54 \mathrm{~F} 50$

\section{Piotr Oprocha}

oprocha@agh.edu.pl

Extended author information available on the last page of the article 


\section{Introduction}

When we perform numerical simulations, it is not completely certain whether sequences generated on the computer screen reflect real dynamical behavior. Moreover, we may also feel unsure when it comes to whether or not every trajectory can be revealed in these simulations. This was the motivation to investigate in the literature the notions of shadowing and inverse-shadowing properties.

The concept of shadowing was introduced independently in the works of Anosov [2] and Bowen [5]. Over the years, many various types of shadowing have been defined. The most important of them include periodic shadowing (tracing periodic pseudoorbits by periodic orbits), inverse shadowing (tracing real orbits by pseudo-orbits produced by methods from a given class of methods), bi-shadowing (combination of shadowing and inverse shadowing) and s-limit shadowing (tracing pseudo-orbits by real orbits with an additional assumption stating that we also properly approximate limit sets by pseudo-orbits with increasing accuracy). In this article, we consider all the kinds of shadowing mentioned above. For the detailed approach to various concepts of shadowing we refer the reader to the monographs [24,26] and (more recent one) [29].

It is quite challenging and often investigated problem to verify whether a given property is generic in a space of maps under consideration, equipped, at least, with the $\mathscr{C}^{0}$-topology; we recall that a property of elements of a topological space $S$ is said to be generic, if it is satisfied on some residual subset of $S$, i.e., on a set that includes a countable intersection of open and dense subsets of $S$. In particular, many scientific works were devoted to genericity of the (usual) shadowing property and its other variants. For example, Yano in [32] proved that shadowing is generic in the space of homeomorphisms of the unit circle and Odani in [22] extended this result to the case of all smooth manifolds of the dimension at most 3. Further outcome was presented in [28], where Pilyugin and Plamenevskaya proved genericity of shadowing for homeomorphisms on any smooth compact manifold without boundary. It has been also showed in [11] and [12] that both shadowing and periodic shadowing are generic in the space of homeomorphisms on a compact smooth manifold, with or without boundary. The same conclusion was confirmed in [13] for some kind of the inverse shadowing property. For an accessible survey of various results of this type, the reader is referred to [27] (see also [1]).

When it comes to the case of non-invertible dynamical systems, one of the first theorem appeared in [18] for continuous maps on an interval. Genericity theorems for shadowing, periodic shadowing and inverse shadowing (with respect to some classes of continuous methods), in the space of all continuous (surjective) functions of a compact topological manifold that admits a decomposition, were proved in [14]. The next results were obtained in [16], where it was showed that shadowing is generic, as well as $s$-limit shadowing is dense in the space of continuous (surjective) maps of any compact topological manifold. At this point it is also worth to mention the results for continuous functions on a Cantor set, showing that in this case there is a residual set of maps which are conjugate with each other and have the shadowing property (see [4] and references therein). It is clear that a special geometric structure of the Cantor set was crucial for such results. Indeed, when proving genericity, geometric structure 


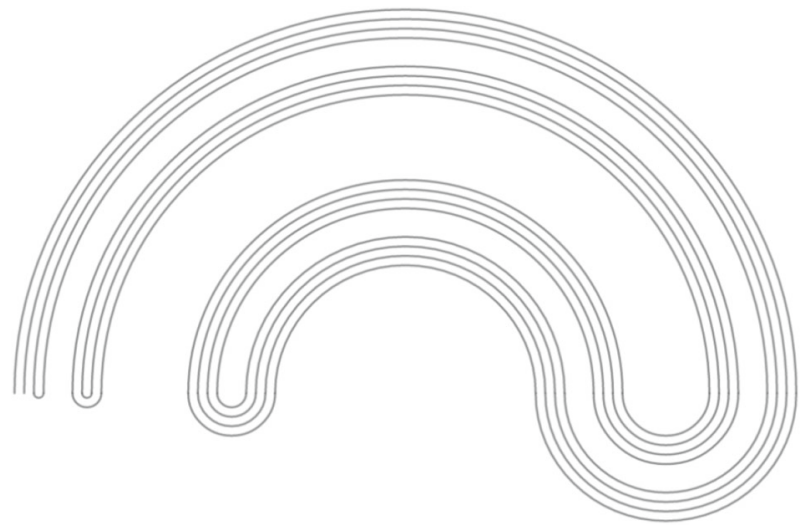

Fig. 1 The Knaster bucket handle

of the space was one of the most important tools; it is enough to mention a novel idea from [28], where the authors used decompositions for the first time, or from [15], where the utility of retractions was demonstrated.

In the first section of the paper we prove that the periodic $\mathscr{T}_{S}$-bi-shadowing property, where $\mathscr{T}_{S}$ means some class of continuous methods, is generic as well as the s-limit shadowing property is dense in the space of all continuous maps (and all continuous surjective maps) of any topological graph. To prove these results we elaborate on a kind of one-dimensional covering relations. In fact, slight modifications of this method are the main tools throughout the paper.

In the second section we prove that $\mathscr{T}_{S}$-bi-shadowing property is generic as well as the $s$-limit shadowing property is dense in the class of all continuous functions defined on any dendrite. Let us mention here that genericity of the (usual) shadowing property on dendrites was recently proved in [6]. In our proof we use a slightly different argument, motivated mainly by the methodology developed for graphs.

In the last section of our work we consider chainable continua that can be well approximated by arcs from the inside. We prove that $\mathscr{T}_{S}$-bi-shadowing property is generic in the class of maps defined on any chainable continuum that is retractable to an arc contained in it. The examples of continua with this property include the $\sin (1 / x)$-curve and the Knaster bucket handle continuum (see Fig. 1 for a sketch of this continuum).

At the end we would like to emphasize that most techniques presented in proofs can be considered (up to some extent) as implementation of Zgliczyński's and Gidea's method of covering relations [34] in its simplest (one-dimensional) form (see also [33]).

\section{Preliminaries}

In this section we present some basic notation that is used throughout the paper. We also recall some basic notions related to the shadowing property and its other variants. 
Additional (more specific) definitions appear successfully in the paper as they are needed.

\subsection{Basic Notation}

The set of all positive integer numbers we denote by $\mathbb{N}$. For any subset $A$ of a given metric space $X$ and any $\varepsilon>0$, by $B_{\varepsilon}(A)$ we mean the $\varepsilon$-envelope of $A$, which contains all the points with the distance from $A$ less than $\varepsilon$. If $R: X \rightarrow Y$, where $Y$ is a subspace of $X$, is a continuous map satisfying $R(x)=x$ for any $x \in Y$, then we call $R$ a retraction onto $Y$.

Let $X$ be a (non-degenerate) continuum, i.e., a compact and connected metric space, possessing more than one point. By a curve in $X$ we mean the image of a continuous map $\gamma$ from the closed unit interval $[0,1]$ into $X$. Then we call $\gamma$ a parametrization of the curve $\gamma([0,1])$.

A curve $J=\gamma([0,1])$ is an arc, if the map $\gamma$ is injective. In this case we call $\gamma(0)$ and $\gamma(1)$ the endpoints of $J$ as well as we denote by $\operatorname{End}(J)$ the set $\{\gamma(0), \gamma(1)\}$.

If $J$ is a curve with a parametrization $\gamma$ such that $\left.\gamma\right|_{(0,1)}$ is injective and $\gamma(0)=$ $\gamma(1)$, then $J$ is called a simple loop or a simple closed curve. Note that, equivalently, a simple loop is the image of a continuous injective map from the unit circle into $X$.

\subsection{Shadowing}

Let $(X, d)$ be a compact metric space. Denote by $C(X)$ the collection of all continuous maps on $X$ endowed with the metric $\rho(f, g)=\sup _{x \in X} d(f(x), g(x))$ for $f, g \in$ $C(X)$. It is well known that $\rho$ is complete and if we denote by $S(X)$ the subset of $C(X)$ consisting of all surjective maps, then $\rho$ restricted to $S(X)$ remains a complete metric. The topology induced by $\rho$ on $C(X)$ (or on $S(X)$ ) is called the $\mathscr{C}^{0}$-topology.

If $f \in C(X)$, then the pair $(X, f)$ is a dynamical system. The orbit of a point $x \in X$ is the sequence $\left\{x_{n}\right\}_{n=0}^{\infty} \subset X$ defined in the following way: $x_{0}=x$ and $x_{n+1}=f\left(x_{n}\right)$ for all integer $n \geq 0$.

Let $f \in C(X)$ and $\delta>0$. A sequence $\left\{x_{n}\right\}_{n=0}^{\infty} \subset X$ is a $\delta$-pseudo-orbit of $f$ if $d\left(f\left(x_{n}\right), x_{n+1}\right)<\delta$ for every integer $n \geq 0$. If, additionally, the sequence $\left\{x_{n}\right\}$ is periodic, i.e., there exists $T>0$ such that $x_{n+T}=x_{n}$ for all integer $n \geq 0$, then it is a periodic $\delta$-pseudo-orbit; in this case we call $T$ a period of $\left\{x_{n}\right\}$ and we also say that $\left\{x_{n}\right\}$ is $T$-periodic. If a $\delta$-pseudo-orbit $\left\{x_{n}\right\}$ satisfies the condition

$$
\lim _{n \rightarrow \infty} d\left(f\left(x_{n}\right), x_{n+1}\right)=0
$$

then it is a limit $\delta$-pseudo-orbit. Clearly, each orbit of $f$ is a (limit) $\delta$-pseudo-orbit of $f$ for any $\delta>0$.

Now, we are ready to present the concepts of shadowing, periodic shadowing and $s$-limit shadowing, which are the main properties studied in this paper.

Definition 1 A map $f \in C(X)$ has the shadowing property if for every $\varepsilon>0$ there exists $\delta>0$ satisfying the following condition: any $\delta$-pseudo-orbit $\left\{y_{n}\right\}$ of $f$ is $\varepsilon$-traced 
by some orbit $\left\{x_{n}\right\}$ of $f$, i.e., $d\left(x_{n}, y_{n}\right)<\varepsilon$ for every integer $n \geq 0$. If, in addition, each periodic $\delta$-pseudo-orbit $\left\{y_{n}\right\}$ (or each limit $\delta$-pseudo-orbit $\left\{y_{n}\right\}$, respectively) is $\varepsilon$-traced by a periodic orbit $\left\{x_{n}\right\}$ with the same period (or is $\varepsilon$-traced by an orbit $\left\{x_{n}\right\}$ and $d\left(x_{n}, y_{n}\right) \rightarrow 0$ as $n \rightarrow \infty$, respectively), then we say that $f$ has the periodic shadowing property (or the s-limit shadowing property, respectively).

In the next definition $X^{\mathbb{N}}$ denotes the space of all one-sided sequences $\left\{x_{n}\right\}_{n=0}^{\infty} \subset X$, endowed with the Tikhonov product topology which makes $X^{\mathbb{N}}$ a compact metrizable space.

Definition 2 Let $f \in C(X)$ and $\delta>0$. By a $\delta$-method for $f$ we mean a map $\chi: X \rightarrow$ $X^{\mathbb{N}}$ such that for every $x \in X$ the sequence $\chi(x)$ is a $\delta$-pseudo-orbit of $f$ with $\chi(x)_{0}=x$ (then we call $\chi(x)$ a $\delta$-pseudo-orbit produced by $\left.\chi\right)$.

We say that a family $\mathscr{T}=\left\{\mathscr{T}_{f, \delta}: \delta>0\right\}$, consisting of classes $\mathscr{T}_{f, \delta}$ of selected $\delta$-methods for $f$, is complete, if $\mathscr{T}_{f, \delta} \neq \emptyset$ for every $\delta>0$.

In the definition below we present a few other concepts of shadowing: the so-called (periodic) $\mathscr{T}$-inverse shadowing and (periodic) $\mathscr{T}$-bi-shadowing.

Definition 3 Let $f \in C(X)$ and $\mathscr{T}=\left\{\mathscr{T}_{f, \delta}: \delta>0\right\}$ be a complete family of classes $\mathscr{T}_{f, \delta}$ of $\delta$-methods for $f$. We say that $f$ has the $\mathscr{T}$-inverse-shadowing property (or $\mathscr{T}$-bi-shadowing property, respectively) if for every $\varepsilon>0$ there exists $\delta>0$ such that for every $\delta$-method $\chi \in \mathscr{T}_{f, \delta}$ the following condition holds: any orbit of $f$ (or any $\delta$-pseudo-orbit of $f$, respectively) is $\varepsilon$-traced by some $\delta$-pseudo-orbit that is produced by $\chi$ (or is $\varepsilon$-traced by some orbit of $f$ as well as by some $\delta$-pseudo-orbit that is produced by $\chi$ ). If, additionally, each periodic orbit of $f$ (or each periodic $\delta$-pseudo-orbit of $f$, respectively) with a period $T>0$ is $\varepsilon$-traced by a $T$-periodic $\delta$-pseudo-orbit that is produced by $\chi$ (or is $\varepsilon$-traced by a $T$-periodic orbit of $f$ as well as by a $T$-periodic $\delta$-pseudo-orbit that is produced by $\chi$, respectively), then we say that $f$ has the periodic $\mathscr{T}$-inverse-shadowing property (or the periodic $\mathscr{T}$-bi-shadowing property, respectively).

It is easily seen that a continuous map has the $\mathscr{T}$-bi-shadowing property if and only if it has both the shadowing and the $\mathscr{T}$-inverse-shadowing properties.

For further considerations we will use a complete family of classes of continuous $\delta$-methods that was introduced in [25] (for homeomorphisms) and investigated in [16] (for continuous maps). It is presented in the following Remark.

Remark 4 Let $f \in C(X)$ and $\delta>0$. Denote by $\mathscr{T}_{A, f, \delta}$ the class of all possible $\delta$-methods for $f$. We define the following class of continuous $\delta$-methods for $f$ :

$$
\begin{gathered}
\mathscr{T}_{S, f, \delta}=\left\{\chi \in \mathscr{T}_{A, f, \delta}: \text { there exists a sequence of maps } \psi_{n} \in C(X)\right. \text { satisfying } \\
\left.\rho\left(\psi_{n}, f\right)<\delta \text { such that } \chi(x)_{n+1}=\psi_{n}\left(\chi(x)_{n}\right) \text { for } x \in X \text { and integer } n \geq 0\right\} .
\end{gathered}
$$

By definition, the family $\mathscr{T}_{S}=\left\{\mathscr{T}_{S, f, \delta}: \delta>0\right\}$ is complete. 


\section{Various Shadowing Properties on Topological Graphs}

\subsection{Preliminary Definitions and Facts}

A topological graph (a graph for short) is a continuum $G$ such that there is a onedimensional simplicial complex $K$ whose geometric carrier is homeomorphic to $G$. This way we can view each topological graph as a continuum embedded in $\mathbb{R}^{3}$ (see, e.g., [19]), and hence we can always consider $G$ to be equipped with a geodesic metric $d$ that is induced from the Euclidean structure in $\mathbb{R}^{3}$ onto an associated simplicial complex $K$. In other words, the distance between two points is equal to the length of the shortest path connecting them. By the definition, each graph $G$ can be represented as the union of a family $\mathscr{I}=\left\{I_{i}\right\}_{i=1}^{n}$, where each set $I_{i}$ is an arc (in practice, a line segment in $\mathbb{R}^{3}$ ) and $\left|I_{j} \cap I_{k}\right| \leq 1$ for $j \neq k$, assuming that non-empty intersection is allowed only in the endpoints of the arcs $I_{i}$ for $i \in\{1, \ldots, n\}$.

Let us establish above-described representation for a moment. A point $x \in G$ is called a vertex of $G$ if it is an endpoint of some arc $I_{i}$.

By the star of a vertex $x \in G$ we mean the set $S(x)=\bigcup\left\{I_{i} \in \mathscr{I}: x \in I_{i}\right\}$. For any point $x \in G$ define the valence of $x$, denoted by $\operatorname{val}(x)$, in the following way: if $x$ is a vertex of $G$ then $\operatorname{val}(x)$ is equal to the number of connected components of the set $S(x) \backslash\{x\}$, and $\operatorname{val}(x)=2$ otherwise. If $\operatorname{val}(x)=1$ (or $\operatorname{val}(x)>2$, respectively), then we call $x$ an endpoint of $G$ (or a branch point of $G$, respectively). The sets of all endpoints and all branch points of $G$ are denoted by $\operatorname{End}(G)$ and $\operatorname{Br}(G)$, respectively. It is not hard to see that these definitions are independent of the choice of a representation of $G$.

All of the above concepts are illustrated in the example shown in Fig. 2.

Before proving the main theorems of this section, we need some technical lemmas. The first two will be helpful in proving the bi-shadowing property and its periodic version. Their proofs are, in fact, easy exercises, which we leave to the reader.

Lemma 5 Let $G$ be a graph and $f \in C(G)$. If $S, V \subset G \backslash \operatorname{Br}(G)$ are arcs such that $V \subset f(S)$ and $f(S)$ does not contain a simple loop, then there exists an arc $S^{*} \subset S$ such that $f\left(S^{*}\right)=V$.

Lemma 6 Let $G$ be a graph and $f \in C(G)$. If $S_{0}, \ldots S_{T-1} \subset G \backslash \operatorname{Br}(G)$ are arcs such that each set $f\left(S_{i}\right)$ has no simple loop as well as $S_{i+1} \subset f\left(S_{i}\right)$ for all $i \in$ $\{0, \ldots, T-2\}$ and $S_{0} \subset f\left(S_{T-1}\right)$, then there exists a point $x \in S_{0}$ such that $f^{T}(x)=x$ and $f^{i}(x) \in S_{i}$ for all $i \in\{0, \ldots, T-1\}$.

In order to formulate the next lemma, which gives some sufficient tools for proving the main results of this section, let us denote by $\alpha_{G}$ the minimal distance between two different points of the set $\operatorname{End}(G) \cup \operatorname{Br}(G)$, i.e.,

$$
\alpha_{G}=\min \{d(a, b): a, b \in \operatorname{End}(G) \cup \operatorname{Br}(G), a \neq b\} .
$$

If there is a simple loop in $G$, then we define

$$
\beta_{G}=\min \{\operatorname{diam} C: C \subset G \text { is a simple loop }\},
$$




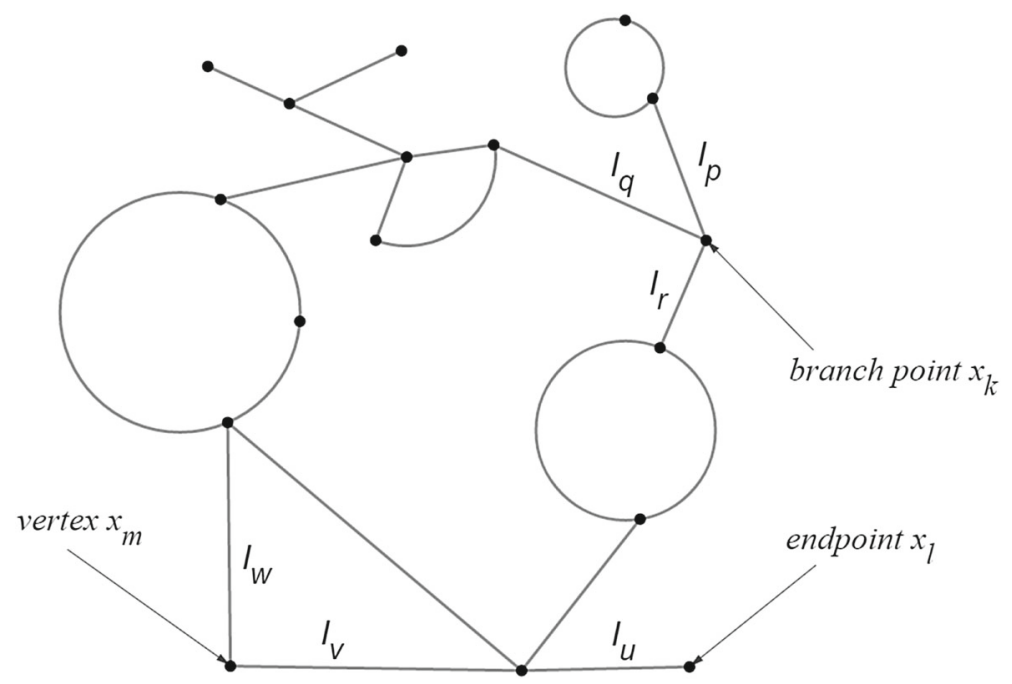

Fig. 2 Sample representation of a graph

and we put $\beta_{G}=\operatorname{diam} G$ otherwise. Finally, we define

$$
\Gamma(G)=\min \left\{\alpha_{G}, \beta_{G}\right\} .
$$

Lemma 7 Let $G$ be a graph, $f \in C(G)$ and $\varepsilon>0$. Let $\left\{U_{i}\right\}_{i=1}^{n}$ be a cover of $G$ consisting of pairwise distinct arcs satisfying

$$
\max \left\{\operatorname{diam} U_{i}, \operatorname{diam} f\left(U_{i}\right)\right\}<\min \{\varepsilon, \Gamma(G) / 3\},
$$

for which the following implication holds:

$$
U_{i} \cap U_{j} \neq \varnothing \Longrightarrow \text { Int } U_{i} \cap \operatorname{Int} U_{j} \neq \emptyset \text {. }
$$

Denote by $\left\{V_{i, j}\right\}_{j=1}^{k(i)}$ for $i \in\{1, \ldots, n\}$ the maximal (in the sense of the number of elements) subfamilies of $\left\{U_{s}\right\}_{s=1}^{n}$ such that

$$
f\left(U_{i}\right) \subset \operatorname{Int} \bigcup_{j=1}^{k(i)} V_{i, j} \text { and } f\left(U_{i}\right) \cap \operatorname{Int} V_{i, j} \neq \varnothing
$$

for each $i \in\{1, \ldots, n\}, j \in\{1, \ldots, k(i)\}$. Under the above assumptions, there exist pairwise disjoint arcs $S_{i, 1}, \ldots, S_{i, k(i)}, i \in\{1, \ldots, n\}$, such that $S_{i, j} \subset \operatorname{Int} U_{i}$ (hence each set $S_{i, j}$ has no branch points), a constant $\xi>0$ and a map $g \in C(G)$ with $\rho(f, g)<\varepsilon$, such that for every $i \in\{1, \ldots, n\}$ the following conditions are satisfied:

(i) the map $\left.g\right|_{S_{i, j}}$ is injective for every $j \in\{1, \ldots, k(i)\}$,

(ii) $g\left(U_{i}\right)=\bigcup_{j=1}^{k(i)} g\left(S_{i, j}\right)$, 
(iii) $B_{\xi}\left(\bigcup_{l=1}^{k(\psi(i, j))} S_{\psi(i, j), l}\right) \subset g\left(S_{i, j}\right)$ for $j \in\{1, \ldots, k(i)\}$ and each set $\overline{B_{\xi}\left(S_{\psi(i, j), l}\right)}$ is an arc containing no branch points,

(iv) $B_{\xi}\left(g\left(U_{i}\right)\right) \subset \bigcup_{j=1}^{k(i)} V_{i, j}$,

where $\psi(i, j)$ is the unique index such that $V_{i, j}=U_{\psi(i, j)}$.

Proof Fix any cover $\left\{U_{i}\right\}_{i=1}^{n}$ of $G$ satisfying required properties and note that intersection of its two different members is an arc, when non-empty.

At first, we claim that for every $i \in\{1, \ldots, n\}$ and $j \in\{1, \ldots k(i)\}$ there exists an arc $W_{i, j} \subset$ Int $V_{i, j} \cup\left(\operatorname{Br}(G) \cap V_{i, j}\right) \subset V_{i, j}$ satisfying the following conditions:

$$
f\left(U_{i}\right) \subset \operatorname{Int} \bigcup_{j=1}^{k(i)} W_{i, j}, \quad f\left(U_{i}\right) \cap \operatorname{Int} W_{i, j} \neq \emptyset, \quad \psi(i, j)=\psi(k, l) \Rightarrow W_{i, j}=W_{k, l} .
$$

Indeed, for any $i \in\{1, \ldots, n\}$ take

$$
\alpha_{i}=\min \left\{\operatorname{diam}\left(V_{i, j} \cap V_{i, k}\right): V_{i, j} \cap V_{i, k} \neq \emptyset\right\},
$$

which, by (3.2), is a positive constant. We also know that $\bigcup_{j=1}^{k(i)} V_{i, j}$ is a subgraph of $G$ containing, by (3.1), at most one point form the set $\operatorname{End}(G) \cup \operatorname{Br}(G)$. Define

$$
\beta_{i}=\min \left\{\operatorname{dist}\left(f\left(U_{i}\right), z\right): z \in \operatorname{End}\left(\bigcup_{j=1}^{k(i)} V_{i, j}\right) \backslash \operatorname{End}(G)\right\}
$$

and note that by compactness of $f\left(U_{i}\right)$ we have $\beta_{i}>0$. Now choose a collection of points $x_{i, j}$ for $i \in\{1, \ldots, n\}$ and $j \in\{1, \ldots, k(i)\}$, such that $x_{i, j} \in f\left(U_{i}\right) \cap \operatorname{Int} V_{i, j}$. Put

$$
\gamma=\min _{i, j}\left\{\operatorname{dist}\left(x_{i, j}, \operatorname{End}\left(V_{i, j}\right) \backslash \operatorname{End}(G)\right)\right\}>0
$$

and

$$
\eta=\min _{i}\left\{\min \left\{\alpha_{i} / 3, \beta_{i} / 3, \gamma / 3\right\}\right\}>0
$$

Then for each $i \in\{1, \ldots, n\}$ and $j \in\{1, \ldots, k(i)\}$ take an arc $W_{i, j} \subset$ Int $V_{i, j} \cup$ $\left(\operatorname{Br}(G) \cap V_{i, j}\right) \subset V_{i, j}$ such that $\operatorname{End}(G) \cap V_{i, j}=\operatorname{End}(G) \cap W_{i, j}$ and all connected components of the set $V_{i, j} \backslash W_{i, j}$ have diameters equal to $\eta$.

Consequently, one can easily check that

$$
x_{i, j} \in f\left(U_{i}\right) \cap \operatorname{Int} W_{i, j} \neq \varnothing \text { and } f\left(U_{i}\right) \subset \operatorname{Int} \bigcup_{j=1}^{k(i)} W_{i, j} .
$$

By the choice of $\eta$, if $V_{i, j}=V_{k, l}$ then $W_{i, j}=W_{k, l}$. Summing up, the condition (3.3) holds. 
Since the sets $W_{i, j}$ depend only on $\eta$ and their supersets $U_{\psi(i, j)}=V_{i, j}$, we obtain a collection of $\operatorname{arcs}\left\{R_{s}\right\}_{s=1}^{n}$ such that $R_{\psi(i, j)}=W_{i, j} \subset U_{\psi(i, j)}$. Observe that if we decrease $\eta$ then we, in fact, decrease $\operatorname{diam}\left(V_{i, j} \backslash W_{i, j}\right)$, so doing this, if necessary, we easily ensure also that the following implication holds:

$$
f\left(U_{i}\right) \cap \operatorname{Int} V_{i, j} \neq \emptyset \Longrightarrow f\left(R_{i}\right) \cap \text { Int } W_{i, j} \neq \emptyset \text {. }
$$

Hence we can find pairwise distinct points $z_{i, j} \in \operatorname{Int} R_{i}$ for $j \in\{1, \ldots, k(i)\}$ such that $f\left(z_{i, j}\right) \in$ Int $W_{i, j}$. In particular, we can construct arcs

$$
S_{i, 1}, \ldots, S_{i, k(i)}, S_{i, 1}^{*}, \ldots, S_{i, k(i)}^{*} \text { for } i \in\{1, \ldots, n\},
$$

such that

$$
\begin{aligned}
S_{i, j} & \subset \operatorname{Int} S_{i, j}^{*} \subset S_{i, j}^{*} \subset \operatorname{Int} R_{i} \backslash \operatorname{End}(G) \subset U_{i} \text { and } \\
f\left(S_{i, j}^{*}\right) & \subset \operatorname{Int} W_{i, j} \subset U_{\psi(i, j)} .
\end{aligned}
$$

Note that each set Int $R_{i}$ does not contain branch points of $G$, so each set $S_{i, j}^{*}$ does not contain them as well. Clearly, we may also assume that all the $\operatorname{arcs} S_{i, j}^{*}$ are pairwise disjoint. Put

$$
\xi:=1 / 2 \cdot \min _{i, j}\left\{\operatorname{dist}\left(S_{i, j}^{*}, \operatorname{End}\left(R_{i}\right) \cup\left(\operatorname{Br}(G) \cap R_{i}\right)\right\}>0,\right.
$$

and note that all the sets $\overline{B_{\xi}\left(S_{i, j}^{*}\right)}$ are arcs with no branch points. We can define a map $g \in C(G)$ by putting on each set $S_{i, j}$ an affine map with the image equal to $W_{i, j}$ and taking

$$
g(x)=f(x) \text { for any } x \in G \backslash \bigcup_{i=1}^{n} \bigcup_{j=1}^{k(i)} \operatorname{Int} S_{i, j}^{*}
$$

By (3.5) we can define $g$ on connected components of the sets Int $S_{i, j}^{*} \backslash S_{i, j}$ in such a way that $g$ is continuous, and the following conditions are satisfied:

$$
g\left(S_{i, j}\right)=g\left(S_{i, j}^{*}\right)=W_{i, j}=R_{\psi(i, j)} \subset V_{i, j}=U_{\psi(i, j)} \text { and }\left.g\right|_{S_{i, j}} \text { is injective }
$$

for $i \in\{1, \ldots, n\}$ and $j \in\{1, \ldots, k(i)\}$. Since the diameter of each $U_{i}$ is less than $\varepsilon$, we conclude that $\rho(f, g)<\varepsilon$, and hence the condition (i) holds.

Recall that $f\left(S_{s, t}^{*}\right) \subset$ Int $W_{s, t} \subset V_{s, t}$ by (3.5), and therefore if $U_{i} \cap S_{s, t}^{*} \neq \varnothing$ then $f\left(U_{i}\right) \cap V_{s, t} \neq \emptyset$ and hence, by (3.3), there exists $1 \leq j \leq k(i)$ such that $W_{s, t}=W_{i, j}$. Taking into account the definition of $g,(3.2),(3.3)$ and (3.6), this immediately implies that

$$
g\left(U_{i}\right) \subset f\left(U_{i}\right) \cup \bigcup_{s=1}^{n} \bigcup_{t=1}^{k(s)} g\left(U_{i} \cap S_{s, t}^{*}\right) \subset \operatorname{Int} \bigcup_{j=1}^{k(i)} W_{i, j} \cup \bigcup_{j=1}^{k(i)} W_{i, j}
$$




$$
\subset \bigcup_{j=1}^{k(i)} W_{i, j} \subset \bigcup_{j=1}^{k(i)} \operatorname{Int} V_{i, j} \cup \operatorname{Br}\left(V_{i, j}\right) \subset \operatorname{Int} \bigcup_{j=1}^{k(i)} V_{i, j}
$$

Hence, decreasing $\xi$ if necessary, we see that the condition (iv) holds. Additionally, by (3.6) we have

$$
\bigcup_{j=1}^{k(i)} W_{i, j}=\bigcup_{j=1}^{k(i)} g\left(S_{i, j}\right) \subset g\left(U_{i}\right)
$$

so the condition (ii) is satisfied.

Finally, note that each set $\overline{B_{\xi}\left(S_{i, j}\right)}$ is an arc containing no branch points of $G$. Furthermore, by the definition of $\xi$ and (3.6) we obtain that

$$
B_{\xi}\left(\bigcup_{l=1}^{k(\psi(i, j))} S_{\psi(i, j), l}\right) \subset R_{\psi(i, j)}=g\left(S_{i, j}\right),
$$

which gives the condition (iii), and hence completes the proof.

Remark 8 Denote $S_{i}:=\bigcup_{j=1}^{k(i)} S_{i, j}$. From the statement of Lemma 7 it follows that for each $i \in\{1, \ldots, n\}$ we have

$$
g\left(U_{i}\right)=g\left(S_{i}\right) \supset \bigcup_{j=1}^{k(i)} B_{\xi}\left(S_{\psi(i, j)}\right) \supset \bigcup_{j=1}^{k(i)} S_{\psi(i, j)}
$$

and $S_{i} \subset$ Int $U_{i}$. It is also clear that if $f \in S(G)$ then also $g \in S(G)$, because, by (3.4) and (3.7), we have $f\left(U_{i}\right) \subset g\left(U_{i}\right)$ for every $i \in\{1, \ldots, n\}$.

Example 9 Fix any graph $G$, any map $f \in C(G)$ and any constant $\varepsilon>0$. We can construct a cover satisfying the assumptions of Lemma 7 as follows. At first fix a positive constant $\gamma<\min \{\varepsilon, \Gamma(G) / 3\}$ such that the following implication holds for any points $x, y \in G$ :

$$
d(x, y)<\gamma \Longrightarrow d(f(x), f(y))<\min \{\varepsilon, \Gamma(G) / 3\} .
$$

If $x \in \operatorname{Br}(G)$ then, obviously, $\overline{B_{\gamma / 2}(x)}$ is an $n$-star $(n \geq 3)$ and thus the set $\overline{B_{\gamma / 2}(x)} \backslash\{x\}$ has $n$ connected components $C_{1}, \ldots, C_{n}$, such that each set $\overline{C_{i}}$ is an arc containing $x$ as one of its endpoints. So we include into the cover the following $n-1 \operatorname{arcs}$ :

$$
\overline{C_{i}} \cup \overline{C_{n}} \text { for } i \in\{1, \ldots, n-1\},
$$

which clearly satisfy the condition (3.2). Next, for each $x \in \operatorname{End}(G)$ the set $\overline{B_{\gamma}(x)}$ is an arc and thus we also consider it as a member of the cover. Finally, each connected 


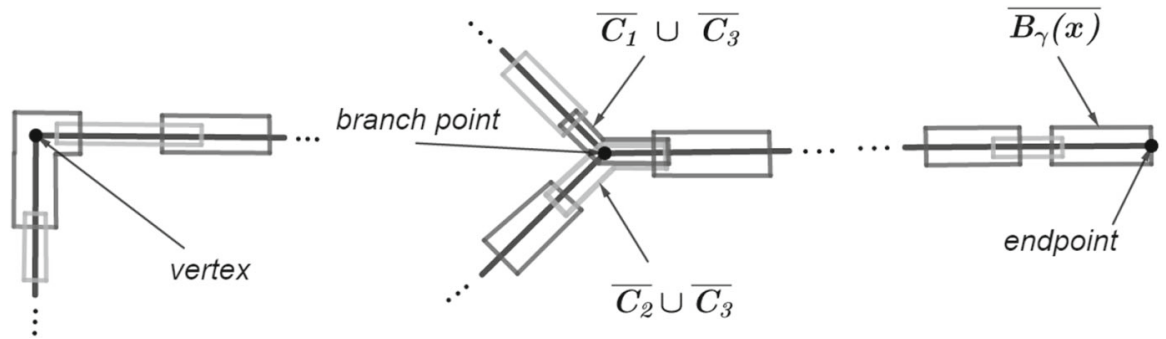

Fig. 3 An illustration of the procedure described in Example 9 in a neighborhood of a vertex, a branch point and an endpoint

component of the set

$$
G \backslash \cup_{x \in \operatorname{End}(G) \cup \operatorname{Br}(G)} B_{\gamma / 4}(x)
$$

is an arc, so it can be easily covered by finite number of arcs with diameters bounded by $\varepsilon$, in such a way that if two arcs intersect, then so do their interiors. Now, it is clear that taking all these sets together we obtain a cover of $G$ satisfying the assumptions of Lemma 7.

The concept of the procedure described in Example 9 is presented in Fig. 3.

\section{2 $\mathscr{T}_{\mathrm{s}}$-bi-Shadowing is $\mathscr{C}^{0}$-Generic on Graphs}

Now, we are ready to prove the first result regarding genericity of shadowing on graphs.

Theorem 10 Let $G$ be a graph. Then for every $\varepsilon>0$ and every map $f \in C(G)$ there exists $\delta>0$ and a map $g \in C(G)$ with $\rho(f, g)<\varepsilon$, such that for every $h \in C(G)$ with $\rho(g, h)<\delta$ and every $\delta$-method $\chi \in \mathscr{T}_{S, h, \delta}$, each (periodic) $\delta$-pseudo-orbit of $h$ is $\varepsilon$-traced by some (periodic) orbit of h as well as by some (periodic) $\delta$-pseudo-orbit of $h$ that is produced by $\chi$. Additionally, if $f \in S(G)$ then also $g \in S(G)$.

Proof Fix any $\varepsilon>0$ and any $f \in C(G)$. Let $\left\{U_{i}\right\}_{i=1}^{n}$ be a cover of $G$ satisfying assumptions of Lemma 7. By definition, for all $i \in\{1, \ldots, n\}$ the set $\bigcup_{j=1}^{k(i)} V_{i, j}$ contains no simple loop. We apply Lemma 7 to this cover and the map $f$ obtaining a map $g \in C(G)$, a constant $\xi>0$ and a collection of arcs $\left\{S_{i, j}\right\}$. Recall that $g \in S(G)$ if $f \in S(G)$ (see Remark 8 for more details). Take any positive number $\delta<\xi / 2$, any map $h \in C(G)$ with $\rho(h, g)<\delta$ and any $\delta$-method $\chi \in \mathscr{T}_{S, h, \delta}$ for $h$, together with the respective functions $\psi_{t} \in C(G)$ with $\rho\left(h, \psi_{t}\right)<\delta$, such that $\chi(x)_{t+1}=\psi_{t}\left(\chi(x)_{t}\right)$ for all $x \in G$ and all $t \geq 0$. Note that then $\rho\left(g, \psi_{t}\right)<2 \delta<\xi$ and $h\left(S_{i, j}\right)$ does not contain a simple loop for any choice of $i \in\{1, \ldots, n\}$ and $j \in\{1, \ldots, k(i)\}$.

Fix a $\delta$-pseudo-orbit $\left\{x_{t}\right\}_{t=0}^{\infty}$ of $h$. Directly from Lemma 7 (iv) we obtain that for every integer $t \geq 0$, if $x_{t} \in U_{i}$ then $x_{t+1} \in \bigcup_{j=1}^{k(i)} V_{i, j}$. Additionally, we can construct sequences $\{\eta(t)\}_{t=0}^{\infty} \subset\{1, \ldots, n\}$ and $\{\xi(t)\}_{t=0}^{\infty} \subset \mathbb{N}$ such that for every integer $t \geq 0$ 
the following conditions are satisfied:

$$
x_{t} \in U_{\eta(t)}, \quad x_{t+1} \in U_{\eta(t+1)} \in\left\{V_{\eta(t), j}\right\}_{j=1}^{k(\eta(t))},
$$

and $\psi(\eta(t), \xi(t))=\eta(t+1)$. Such selection is possible, because by Lemma 7 (iv) we have

$$
x_{t+1} \in B_{\delta}\left(h\left(x_{t}\right)\right) \subset B_{2 \delta}\left(g\left(x_{t}\right)\right) \subset B_{\xi}\left(g\left(U_{\eta(t)}\right)\right) \subset \bigcup_{j=1}^{k(\eta(t))} V_{\eta(t), j} .
$$

Then, by the definition of the sets $S_{i, j}$, we have $S_{\eta(t), \xi(t)} \subset U_{\eta(t)}$ and, by Lemma 7 (iii), we additionally obtain

$$
\begin{aligned}
B_{\xi}\left(S_{\eta(t+1), \xi(t+1)}\right)= & B_{\xi}\left(S_{\psi(\eta(t), \xi(t)), \xi(t+1)}\right) \subset B_{\xi}\left(\bigcup_{l=1}^{k(\psi(\eta(t), \xi(t)))} S_{\psi(\eta(t), \xi(t)), l}\right) \\
& \subset g\left(S_{\eta(t), \xi(t)}\right) .
\end{aligned}
$$

Therefore,

$$
S_{\eta(t+1), \xi(t+1)} \subset h\left(S_{\eta(t), \xi(t)}\right) \text { and } S_{\eta(t+1), \xi(t+1)} \subset \psi_{t}\left(S_{\eta(t), \xi(t)}\right) .
$$

By the above,

$$
\bigcap_{t=0}^{\infty} h^{-t}\left(S_{\eta(t), \xi(t)}\right) \neq \varnothing \text { and } S_{\eta(0), \xi(0)} \cap \bigcap_{t=1}^{\infty}\left(\psi_{t-1} \circ \cdots \circ \psi_{0}\right)^{-1}\left(S_{\eta(t), \xi(t)}\right) \neq \emptyset
$$

which implies that there exist points $y, z \in S_{\eta(0), \xi(0)}$ such that for each $t \geq 0$ we have $h^{t}(y), \chi(z)_{t} \in S_{\eta(t), \xi(t)} \subset U_{\eta(t)}$. In particular,

$$
d\left(h^{t}(y), x_{t}\right) \leq \operatorname{diam}\left(U_{\eta(t)}\right)<\varepsilon \text { and } d\left(\chi(z)_{t}, x_{t}\right) \leq \operatorname{diam}\left(U_{\eta(t)}\right)<\varepsilon
$$

for every integer $t \geq 0$.

Now, assume that $\left\{x_{t}\right\}_{t=0}^{\infty}$ is a periodic $\delta$-pseudo-orbit of $h$, i.e., $x_{t+T}=x_{t}$ for some integer $T>0$ and all $t \geq 0$. It is easy to see that we can then put $\eta(t)=\eta(t+T)$ and setting $\xi(t+T)=\xi(t)$ we obtain

$$
\eta(t+T+1)=\eta(t+1)=\psi(\eta(t), \xi(t))=\psi(\eta(t+T), \xi(t+T)),
$$

so all the previous calculations hold and, consequently, this sequence is good for proving tracing. But then, in particular, we have $S_{\eta(0), \xi(0)}=S_{\eta(T), \xi(T)}$, and hence by Lemma 6 we conclude that there are points $y, z \in S_{\eta(0), \xi(0)}$, such that the orbit of $y$ and the $\delta$-pseudo-orbit $\chi(z)$ are periodic (with a period $T$ ) and both $\varepsilon$-trace $\left\{x_{t}\right\}_{t=0}^{\infty}$. The proof is completed. 
Theorem 11 For any graph $G$, the set of continuous maps possessing the periodic $\mathscr{T}_{S}$-bi-shadowing property is residual in $C(G)$ and in $S(G)$.

Proof Let $\mathscr{X}=C(G)$ or $\mathscr{X}=S(G)$, depending on the case we are proving.

For each $n \in \mathbb{N}$ denote by $A_{n} \subset \mathscr{X}$ the set consisting of maps $g \in \mathscr{X}$ for which there are constants $\delta>0$ such that if $h \in \mathscr{X}$ satisfies $\rho(g, h)<\delta$ and $\chi \in \mathscr{T}_{S, h, \delta}$, then every (periodic) $\delta$-pseudo-orbit of $h$ is $1 / n$-traced by some (periodic) orbit of $h$ and by some (periodic) $\delta$-pseudo-orbit of $h$ that is produced by $\chi$.

We are going to show that each set $A_{n}$ is open in $\mathscr{X}$. Indeed, fix $n \in \mathbb{N}, g \in A_{n}$ and take a respective constant $\delta>0$. We claim that $B_{\delta}(g) \subset A_{n}$. Indeed, for a given $h \in B_{\delta}(g)$ there exists $\eta>0$ such that $B_{\eta}(h) \subset B_{\delta}(g)$. Hence, if $\tilde{h} \in \mathscr{X}$ satisfies $\rho(h, \tilde{h})<\eta$ then $\tilde{h} \in B_{\delta}(g)$, which implies that if we establish $\chi \in \mathscr{T}_{S, \tilde{h}, \eta} \subset \mathscr{T}_{S, \tilde{h}, \delta}$, then every (periodic) $\eta$-pseudo-orbit of $\tilde{h}$, being at the same time a (periodic) $\delta$-pseudoorbit of $\tilde{h}$, is $1 / n$-traced by some orbit of $\tilde{h}$ and by some (periodic) $\eta$-pseudo-orbit of $\tilde{h}$ that is produced by $\chi$. So indeed $h \in A_{n}$, which completes the proof of the openness of $A_{n}$.

On the other hand, by Theorem 10 , the set $A_{n}$ is also dense in $\mathscr{X}$ and therefore $\bigcap_{n} A_{n}$ is a residual subset of $\mathscr{X}$. To finish the proof it is enough to note that, by definition, every map in the set $\bigcap_{n} A_{n}$ has the periodic $\mathscr{T}_{S}$-bi-shadowing property.

\subsection{S-Limit Shadowing is $\mathscr{C}^{0}$-Dense on Graphs}

For the proof of the second result of this section we need the following lemma, which gives a tool that allows us to deal with tracing limit $\delta$-pseudo-orbits with increasing accuracy.

Lemma 12 Let $G$ be a graph, $f \in C(G)$ and $\varepsilon>0$. Let $\left\{U_{i}\right\}_{i=1}^{n}$ be a cover of $G$ consisting of pairwise distinct arcs, such that the conditions (3.1) and (3.2) are satisfied. Furthermore, assume that the arcs $\left\{S_{i, j}\right\}_{j=1}^{k(i)}, i \in\{1, \ldots, n\}$, and a continuous map $g \in C(G)$ are provided by Lemma 7 for the above setting.

Then for any $\gamma>0$ there exists a refinement $\left\{W_{s}\right\}_{s=1}^{m}$ of $\left\{U_{i}\right\}_{i=1}^{n}$ (i.e., each $W_{s}$ is a subset of some $U_{i}$ ), consisting of arcs satisfying the condition (3.2), such that

$\max \left\{\operatorname{diam}\left(W_{s}\right), \operatorname{diam}\left(g\left(W_{s}\right)\right)\right\}<\min \{\gamma, \Gamma(G) / 3\}$ for each $s \in\{1, \ldots, m\}$

and the following conditions hold:

(1) for each $i \in\{1, \ldots, n\}$ we have

$$
\bigcup\left\{W \in\left\{W_{s}\right\}_{s=1}^{m} \mid W \subset U_{i}\right\}=U_{i},
$$

(2) for each $i \in\{1, \ldots, n\}$ and each $j \in\{1, \ldots, k(i)\}$ we have

$$
\bigcup\left\{W \in\left\{W_{s}\right\}_{s=1}^{m} \mid W \subset S_{i, j}\right\}=S_{i, j} .
$$

Proof It is an easy exercise. We leave details to the reader. 
The proof of the following theorem, which is our last result for continuous maps on graphs, follows similar lines to the proof of Theorem 2 in [16], with the main difference that we need to use Lemma 7 to overcome technical difficulties of the considered setting.

Theorem 13 For any graph $G$, the set of continuous maps with the s-limit shadowing property is dense in $C(G)$ and in $S(G)$.

Proof Let $\mathscr{X}=C(G)$ or $\mathscr{X}=S(G)$, depending on the case we are proving.

Let $f \in \mathscr{X}$ and $\varepsilon>0$. Put $\varepsilon_{1}:=\varepsilon$ and $g_{0}=f$. Take $g_{1} \in \mathscr{X}$ and $\xi_{1}>0$ with $\rho\left(g_{0}, g_{1}\right)<\varepsilon_{1}$, as well as a collection of sets $\left\{S_{i, j}^{1}\right\}$ provided by Lemma 7 for some cover $\left\{U_{i}^{1}\right\}$, consisting of sets with sufficiently small diameters, satisfying the conditions (3.1) and (3.2). Take a refinement $\left\{U_{i}^{2}\right\}$ of the cover $\left\{U_{i}^{1}\right\}$ provided by Lemma 12 with $\gamma=\varepsilon_{2} / 16$, where $\varepsilon_{2}:=\min \left\{\xi_{1} / 2, \varepsilon_{1}-\rho\left(g_{0}, g_{1}\right), \varepsilon_{1} / 2\right\}$. Take $g_{2} \in \mathscr{X}$ with $\rho\left(g_{1}, g_{2}\right)<\varepsilon_{2}$, a constant $\xi_{2}<\xi_{1}$ and a collection of sets $\left\{S_{i, j}^{2}\right\}$ by Lemma 7 for the cover $\left\{U_{i}^{2}\right\}$.

Proceeding inductively in this way we obtain sequences $\left\{\varepsilon_{m}\right\},\left\{\xi_{m}\right\},\left\{U_{i}^{m}\right\},\left\{S_{i, j}^{m}\right\}$, $\left\{g_{m}\right\}$ such that

$$
\rho\left(g_{m-1}, g_{m}\right)<\varepsilon_{m}=\min \left\{\xi_{m-1} / 2, \varepsilon_{m-1}-\rho\left(g_{m-2}, g_{m-1}\right), \varepsilon_{m-1} / 2\right\} \leq \varepsilon / 2^{m-1} .
$$

All the properties of the collections of sets $\left\{U_{i}^{m-1}\right\},\left\{U_{i}^{m}\right\},\left\{S_{i, j}^{m-1}\right\}$ and $\left\{S_{i, j}^{m}\right\}$ mentioned above apply accordingly.

Since $\mathscr{X}$ is a complete metric space and $\left\{g_{m}\right\}$ is a Cauchy sequence, we have $g_{m} \rightarrow g \in \mathscr{X}$ when $m \rightarrow \infty$. Observe that

$$
\begin{aligned}
& \rho\left(g_{0}, g\right) \leq \sum_{n=1}^{\infty} \rho\left(g_{n-1}, g_{n}\right) \leq \varepsilon \sum_{n=1}^{\infty} \frac{1}{2^{n-1}}=2 \varepsilon, \\
& \rho\left(g_{m}, g\right) \leq \sum_{n=m}^{\infty} \rho\left(g_{n}, g_{n+1}\right) .
\end{aligned}
$$

To estimate the upper bound in the above formula, observe that for every $k>0$ we have

$$
\begin{aligned}
\sum_{n=m}^{m+k} \rho\left(g_{n}, g_{n+1}\right) & =\sum_{n=m}^{m+k-2} \rho\left(g_{n}, g_{n+1}\right)+\rho\left(g_{m+k-1}, g_{m+k}\right)+\rho\left(g_{m+k}, g_{m+k+1}\right) \\
& \leq \sum_{n=m}^{m+k-2} \rho\left(g_{n}, g_{n+1}\right)+\varepsilon_{m+k} \\
& \leq \sum_{n=m}^{m+k-2} \rho\left(g_{n}, g_{n+1}\right)+\varepsilon_{m+k-1}-\rho\left(g_{m+k-2}, g_{m+k-1}\right)
\end{aligned}
$$




$$
\begin{aligned}
& =\sum_{n=m}^{m+k-3} \rho\left(g_{n}, g_{n+1}\right)+\varepsilon_{m+k-1} \leq \cdots \leq \rho\left(g_{m}, g_{m+1}\right)+\varepsilon_{m+2} \\
& \leq \rho\left(g_{m}, g_{m+1}\right)+\varepsilon_{m+1}-\rho\left(g_{m}, g_{m+1}\right)=\varepsilon_{m+1} \leq \xi_{m} / 2,
\end{aligned}
$$

and therefore

$$
\rho\left(g_{m}, g\right) \leq \xi_{m} / 2
$$

To show that $g$ has the s-limit shadowing property take $\tau>0$ and establish $N>0$ such that $\varepsilon_{N}<\tau$. Take any $\xi_{N} / 2$-limit-pseudo-orbit $\left\{x_{t}\right\}_{t=0}^{\infty}$ of $g$. Then there exists an increasing sequence $\left\{t_{m}\right\}_{m \geq N}\left(t_{N}:=0\right)$ such that $\left\{x_{t}\right\}_{t \geq t_{m}}$ is a $\xi_{m} / 2$-pseudo-orbit of $g$. Since $\rho\left(g_{m}, g\right) \leq \xi_{m} / 2$ for $m \geq N$, our construction (by Lemma 7 and Lemma 12) ensures that there are sequences

$$
\left\{U_{\eta^{m}\left(t_{m}\right)}^{m}, U_{\eta^{m}\left(t_{m}+1\right)}^{m}, \ldots, U_{\eta^{m}\left(t_{m+1}-1\right)}^{m}, U_{\eta^{m}\left(t_{m+1}\right)}^{m}\right\}, m \geq N
$$

satisfying

$$
\begin{aligned}
& U_{\eta^{m+1}\left(t_{m+1}\right)}^{m+1} \subset U_{\eta^{m}\left(t_{m+1}\right)}^{m}, \\
& \quad x_{t} \in U_{\eta^{m}(t)}^{m} \text { for } t \in\left\{t_{m}, t_{m}+1, \ldots, t_{m+1}\right\}
\end{aligned}
$$

and

$$
U_{\eta^{m}(t+1)}^{m} \in\left\{V_{\eta^{m}(t), j}^{m}\right\}_{j=1}^{k^{m}\left(\eta^{m}(t)\right)} \text { for } t \in\left\{t_{m}, t_{m}+1, \ldots, t_{m+1}-1\right\}
$$

It is possible, because to define $U_{\eta^{m+1}\left(t_{m+1}\right)}^{m+1}$ we can take any set $U_{i}^{m+1} \subset U_{\eta^{m}\left(t_{m+1}\right)}^{m}$ of the refining cover, which satisfies $x_{t_{m+1}} \in U_{i}^{m+1}$ (by Lemma 12 there is at least one such set).

By the above construction we obtain that

$$
g_{m-1}\left(U_{\eta^{m}(t)}^{m}\right) \cap \operatorname{Int} U_{\eta^{m}(t+1)}^{m} \neq \emptyset \text { for } t \in\left\{t_{m}, t_{m}+1, \ldots, t_{m+1}-1\right\} .
$$

Note that for $t \in\left\{t_{m}, t_{m}+1, \ldots, t_{m+1}-1\right\}$ we have

$$
d\left(g_{m}\left(x_{t}\right), x_{t+1}\right) \leq d\left(g_{m}\left(x_{t}\right), g\left(x_{t}\right)\right)+d\left(g\left(x_{t}\right), x_{t+1}\right) \leq \xi_{m},
$$

and hence directly by Remark 8 we obtain that

$$
S_{\eta^{m}(t+1)}^{m} \subset \bigcup_{j=1}^{k^{m}\left(\eta^{m}(t)\right)} S_{\psi^{m}\left(\eta^{m}(t), j\right)}^{m} \subset g\left(S_{\eta^{m}(t)}^{m}\right) \quad \text { for } t=t_{m}, t_{m}+1, \ldots, t_{m+1}-1
$$


To finish the construction we are going to show that there is a point $y \in S_{\eta^{N}(0)}^{N}$ such that $d\left(f^{j}(y), x_{j}\right) \rightarrow 0$ if $j \rightarrow \infty$. As the first step, let us verify that

$$
S_{\eta^{m+1}\left(t_{m+1}+1\right)}^{m+1} \subset g\left(S_{\eta^{m}\left(t_{m+1}\right)}^{m}\right) \text { for } m \geq N
$$

Indeed, note that

$$
g_{m}\left(U_{\eta^{m+1}\left(t_{m+1}\right)}^{m+1}\right) \subset g_{m}\left(U_{\eta^{m}\left(t_{m+1}\right)}^{m}\right)=g_{m}\left(S_{\eta^{m}\left(t_{m+1}\right)}^{m}\right)
$$

and by (3.9) we also have

$$
g_{m}\left(U_{\eta^{m+1}\left(t_{m+1}\right)}^{m+1}\right) \cap \operatorname{Int} U_{\eta^{m+1}\left(t_{m+1}+1\right)}^{m+1} \neq \emptyset,
$$

and hence

$$
g_{m}\left(S_{\eta^{m}\left(t_{m+1}\right)}^{m}\right) \cap \operatorname{Int} U_{\eta^{m+1}\left(t_{m+1}+1\right)}^{m+1} \neq \emptyset
$$

By Lemma 12, which was used to define consecutive refinements, there is some $U_{q}^{m+1} \subset S_{\eta^{m}\left(t_{m+1}\right)}^{m}$ from the cover $\left\{U_{i}^{m+1}\right\}$, such that

$$
g_{m}\left(U_{q}^{m+1}\right) \cap \operatorname{Int} U_{\eta^{m+1}\left(t_{m+1}+1\right)}^{m+1} \neq \emptyset
$$

which means that

$$
U_{\eta^{m+1}\left(t_{m+1}+1\right)}^{m+1} \in\left\{V_{q, j}^{m+1}\right\}_{j=1}^{k^{m+1}(q)} .
$$

Then, since $\rho\left(g, g_{m+1}\right)<\xi_{m+1} / 2$ we obtain (by Remark 8) that

$$
S_{\eta^{m+1}\left(t_{m+1}+1\right)}^{m+1} \subset g\left(S_{q}^{m+1}\right) \subset g\left(U_{q}^{m+1}\right) \subset g\left(S_{\eta^{m}\left(t_{m+1}\right)}^{m}\right) .
$$

Hence by (3.10) and (3.11) we see that (recall that $t_{N}=0$ )

$$
S_{\eta^{N}(0)}^{N} \cap \bigcap_{m=N}^{\infty} \bigcap_{j=t_{m}+1}^{t_{m+1}} g^{-j}\left(S_{\eta^{m}(j)}^{m}\right) \neq \emptyset
$$

which implies that there exists a point $y \in S_{\eta^{N}(0)}^{N}$ such that

$$
g^{j}(y) \in S_{\eta^{m}(j)}^{m} \subset U_{\eta^{m}(j)}^{m},
$$

and, as a consequence, we conclude that, if $t_{m}<j \leq t_{m+1}$ then

$$
d\left(x_{j}, g^{j}(y)\right) \leq \operatorname{dist}\left(x_{j}, S_{\eta^{m}(j)}^{m}\right) \leq \operatorname{diam}\left(U_{\eta^{m}(j)}^{m}\right)<\varepsilon_{m} .
$$

The proof is completed. 


\section{Various Shadowing Properties on Dendrites}

\subsection{Preliminary Definitions and Facts}

A continuum $D$ is a (non-degenerate) dendrite, if $D$ is a locally connected set containing no simple loop. It is known (by the results due to Ważewski and Manger, see, e.g., [30]), that $D$ can be embedded in $\mathbb{R}^{2}$, which allows us, as in the case of graphs, to consider a geodesic metric $d$ on $D$ that is induced by the Euclidean structure in $\mathbb{R}^{2}$.

A point $x \in D$ is called an endpoint of $D$, if the set $D \backslash\{x\}$ is connected, as well as is called a branch point of $D$, if the set $D \backslash\{x\}$ has at least three connected components. Similarly as in the case of graphs, we denote by $\operatorname{End}(D)$ and $\operatorname{Br}(D)$ the sets of endpoints and branch points of $D$, respectively.

The following lemma summarizes a few useful properties of dendrites (see Chapter $\mathrm{X}$ in [20] for proofs of these facts).

Lemma 14 Let $D$ be a dendrite and let $Y \subset D$ be a continuum. Then we have the following conclusions.

(1) The set of branch points of $D$ is countable.

(2) An intersection of two connected subsets of D is either empty or connected.

(3) The continuum $Y$ is a dendrite itself, in particular $Y$ is uniquely arcwise connected, i.e., for any two different points $x, y \in D$ there is a unique arc $Z_{x, y}$ joining $x$ with $y$.

(4) For any $x \in X \backslash Y$ there exists a unique point $R(x) \in Y$ such that $R(x)$ belongs to any arc between $x$ and a point $y \in Y$.

(5) If a map $R: X \rightarrow Y$ is a map obtained by extending the above definition by putting $R(x)=x$ for $x \in Y$, then $R$ is continuous (we call it the first point map for $Y$ ).

(6) There exists an increasing sequence of sets $Y_{1} \subset Y_{2} \subset \ldots \subset D$ such that

(i) each set $Y_{i}$ is a tree (i.e., a graph which does not contain a simple loop) and $\lim _{i \rightarrow \infty} Y_{i}=D$ in the Hausdorff distance,

(ii) $Y_{1}=\left\{p_{1}\right\}$ and for each $i \in \mathbb{N}$ the set $\overline{Y_{i+1} \backslash Y_{i}}$ is an arc with an endpoint $p_{i}$ such that $\overline{Y_{i+1} \backslash Y_{i}} \cap Y_{i}=\left\{p_{i}\right\}$,

(iii) the sequence of the first point maps $R_{i}: X \rightarrow Y_{i}$ converges uniformly to the identity map.

We refer the readers not familiar with the theory of dendrites to the monograph [20], where an accessible presentation of this topic can be found.

The following lemma is the main tool for proving the results of this section.

Lemma 15 Let $D$ be a dendrite, $f \in C(D), \varepsilon>0$ and $\gamma<\varepsilon / 8$ be a positive constant such that if $d(x, y)<\gamma$ then $d(f(x), f(y))<\varepsilon / 8$. Let $T \subset D$ be a tree such that $\rho(R, i d)<\gamma$, where $R$ denotes the first point map for $T$. Let $\left\{\tilde{U}_{i}\right\}_{i=1}^{n}$ be a cover of $T$ meeting the assumptions of Lemma 7 for the map $\left.R \circ f\right|_{T} \in C(T)$ and the constant $\varepsilon / 4$. Take respective families of sets $\left\{\tilde{V}_{i, j}\right\}$ and $\left\{S_{i, j}\right\}$ for $i \in\{1, \ldots, n\}, j \in\{1, \ldots, k(i)\}$, provided by Lemma 7 , and put $U_{i}=R^{-1}\left(\tilde{U}_{i}\right)$ (consequently, $V_{i, j}=R^{-1}\left(\tilde{V}_{i, j}\right)$ ). 
Then $\left\{U_{i}\right\}_{i=1}^{n}$ is a cover of $D$ consisting of connected sets with diameters less than $\varepsilon / 2$ as well as there exist a constant $\eta>0$ and a map $g \in C(D)$ with $\rho(f, g)<\varepsilon / 2$, such that for any map $h \in C(D)$, with $\rho(g, h)<\eta$, the following conditions are satisfied for every $i \in\{1, \ldots, n\}$ :

(i) $B_{\eta}\left(h\left(U_{i}\right)\right) \subset \bigcup_{j=1}^{k(i)} V_{i, j}$,

(ii) $\bigcup_{l=1}^{k(\psi(i, j))} S_{\psi(i, j), l} \subset h\left(S_{i, j}\right)$ for $j \in\{1, \ldots, k(i)\}$,

where $\psi(i, j)$ is the unique index such that $V_{i, j}=U_{\psi(i, j)}$.

Proof At first observe that if $R: X \rightarrow Z \subset X$ is a continuous map such that $\rho(R, i d)<\zeta$, then for every set $A \subset Z$ we have

$$
\operatorname{diam}\left(R^{-1}(A)\right) \leq \operatorname{diam}(A)+2 \zeta
$$

Take a constant $\xi>0$ and a map $\tilde{g} \in C(T)$ with $\rho\left(\left.R \circ f\right|_{T}, \tilde{g}\right)<\varepsilon / 4$, provided by Lemma 7 for the map $\left.R \circ f\right|_{T} \in C(T)$, the constant $\varepsilon / 4$ and the cover $\left\{\tilde{U}_{i}\right\}_{i=1}^{n}$ of $T$. Put $g=\tilde{g} \circ R$. Observe that by (4.1) we have

$$
\operatorname{diam}\left(R^{-1}\left(\tilde{U}_{i}\right)\right) \leq \operatorname{diam}\left(\tilde{U}_{i}\right)+2 \gamma<\frac{\varepsilon}{4}+\frac{\varepsilon}{4}=\frac{\varepsilon}{2}
$$

and, consequently,

$$
\begin{aligned}
d(f(x), g(x)) \leq & d(f(x), f(R(x))) \\
& +d(f(R(x)), R(f(R(x))))+d(R(f(R(x))), \tilde{g}(R(x))) \\
< & \frac{\varepsilon}{8}+\gamma+\frac{\varepsilon}{4}<\frac{\varepsilon}{2},
\end{aligned}
$$

which implies that $\max \left\{\operatorname{diam}\left(U_{i}\right)\right\}<\varepsilon / 2$ and $\rho(f, g)<\varepsilon / 2$. It is easily seen that $\left\{U_{i}\right\}_{i=1}^{n}$ is a cover of $D$ consisting of connected sets. Note that we also have $R\left(U_{i}\right)=U_{i} \cap T=\tilde{U}_{i}$.

Since $\left.g\right|_{T}=\tilde{g}$, by Lemma 7 (iii) we have that

$$
B_{\xi}^{T}\left(\bigcup_{l=1}^{k(\psi(i, j))} S_{\psi(i, j), l}\right) \subset g\left(S_{i, j}\right)
$$

for $j \in\{1, \ldots, k(i)\}$, where $\psi(i, j)$ is the unique index such that $\tilde{V}_{i, j}=\tilde{U}_{\psi(i, j)}$ (hence $\left.V_{i, j}=U_{\psi(i, j)}\right)$ and $B_{\xi}^{T}(\cdot)$ is a $\xi$-envelope in $T$, i.e., $B_{\xi}^{T}(\cdot)=T \cap B_{\xi}(\cdot)$. By Lemma 7 (iv) we also obtain that $B_{\xi}^{T}\left(\tilde{g}\left(\tilde{U}_{i}\right)\right) \subset \bigcup_{j=1}^{k(i)} \tilde{V}_{i, j}$, which leads to the following sequence of inclusions:

$$
B_{\xi}\left(g\left(U_{i}\right)\right) \subset R^{-1}\left(B_{\xi}^{T}\left(\tilde{g}\left(\tilde{U}_{i}\right)\right)\right) \subset \bigcup_{j=1}^{k(i)} R^{-1}\left(\tilde{V}_{i, j}\right)=\bigcup_{j=1}^{k(i)} V_{i, j}
$$


Indeed, it is enough to note that if $W \subset D$ is a connected set (hence also arcwise connected) such that $W \cap T \neq \emptyset$, then $R(W)=W \cap T$.

Take sufficiently small $\eta<\xi / 2$, so that if $d(x, y)<\eta$ then $d(R(x), R(y))<\xi / 2$. Fix any map $h \in C(D)$ such that $\rho(h, g)<\eta$. By (4.3) we obtain

$$
B_{\eta}\left(h\left(U_{i}\right)\right) \subset B_{\xi}\left(g\left(U_{i}\right)\right) \subset \bigcup_{j=1}^{k(i)} V_{i, j}
$$

By Lemma 7 (iii) each set $\overline{B_{\xi}^{T}\left(S_{\psi(i, j), l}\right)}$ is an arc, and therefore, by (4.2), there are points $a, b \in S_{i, j}$ such that

$$
B_{\xi}^{T}\left(S_{\psi(i, j), l}\right)=Z_{g(a), g(b)} \subset T .
$$

Indeed, it is enough to take any points $a$ and $b$, which are mapped by $g$ onto different endpoints of the $\operatorname{arc} \overline{B_{\xi}^{T}\left(S_{\psi(i, j), l}\right)}$. Note also that the $\overline{\operatorname{arc}} \overline{B_{\xi}^{T}\left(S_{\psi(i, j), l}\right) \backslash S_{\psi(i, j), l}} \subset T$ has two connected components, both of them being arcs having diameters equal to $\xi$. By the definition of $\eta$, since $d(g(a), h(a))<\eta$ and $d(g(b), h(b))<\eta$ we have $d(g(a), R(h(a)))<\xi / 2$ and $d(g(b), R(h(b)))<\xi / 2$. This implies that the unique arc $Z_{h(a), h(b)}$ joining $h(a)$ with $h(b)$ have to pass through both connected components of $\overline{B_{\xi}^{T}\left(S_{\psi(i, j), l}\right)} \backslash B_{\xi / 2}^{T}\left(S_{\psi(i, j), l}\right)$ and therefore

$$
S_{\psi(i, j), l} \subset Z_{h(a), h(b)} \subset h\left(S_{i, j}\right) .
$$

The proof is completed.

Remark 16 Let us consider a dendrite $D$ shown in Fig. 4. We take a tree $T \subset D$ consisting of the only horizontal segment $T_{1}$ of $D$ as well as the first two (counting from the right) vertical ones $T_{2}$ and $T_{3}$. The figure presents a concept of construction of covers $\left\{\tilde{U}_{i}\right\}$ and $\left\{U_{i}\right\}$ satisfying the assumptions of Lemma 15.

Remark 17 From the statement of Lemma 15 it follows that for each $i \in\{1, \ldots, n\}$ and every map $h \in C(D)$ such that $\rho(g, h)<\eta$ we have

$$
h\left(S_{i}\right) \supset \bigcup_{j=1}^{k(i)} S_{\psi(i, j)}
$$

and $S_{i} \subset U_{i}$, where $S_{i}:=\bigcup_{j=1}^{k(i)} S_{i, j}$.

\section{2 $\mathscr{T}_{\mathrm{s}}$-bi-Shadowing is $\mathscr{C}^{0}$-Generic on Dendrites}

One of the aims of this section is to prove a result similar to Theorem 11. Unfortunately, we do not have as good covering property as in the case of graphs. Because of that 


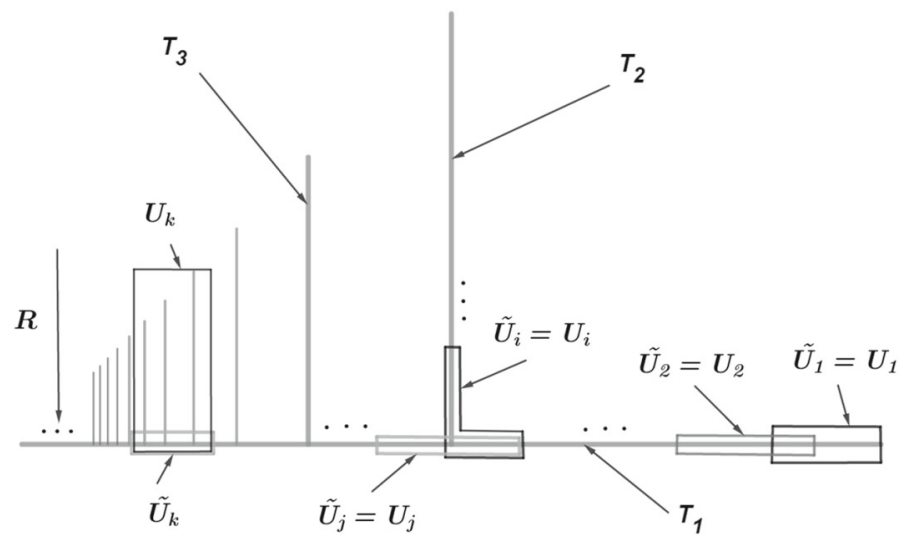

Fig. 4 The idea of a construction of covers satisfying the assumptions of Lemma 15

difficulty, we are not able to prove (using this method) periodic version of the bishadowing property in the case of dendrites. Additionally, proposed approach does not cover the case of surjective mappings.

Theorem 18 Let $D$ be a dendrite. Then for every $\varepsilon>0$ and every map $f \in C(D)$ there is $\delta>0$ and a map $g \in C(D)$ with $\rho(f, g)<\varepsilon$, such that for every $h \in C(D)$ with $\rho(g, h)<\delta$ and every $\delta$-method $\chi \in \mathscr{T}_{S, h, \delta}$, each $\delta$-pseudo-orbit of h is $\varepsilon$-traced by some orbit of $h$ as well as by some $\delta$-pseudo-orbit of $h$ that is produced by $\chi$.

Proof Fix any $\varepsilon>0$ and any $f \in C(D)$. Take a cover $\left\{U_{i}\right\}_{i=1}^{n}$ of $D$, consisting of pairwise distinct connected sets with diameters less than $\varepsilon / 2$, a map $g \in C(D)$ with $\rho(f, g)<\varepsilon / 2$ and a constant $\eta>0$, provided by Lemma 15 for properly chosen tree $T \subset D$ and a cover $\left\{\tilde{U}_{i}\right\}_{i=1}^{n}$ of $T$. Put $\delta=\eta / 2$. Fix any $h \in C(D)$ with $\rho(h, g)<\delta$ and any $\delta$-method $\chi \in \mathscr{T}_{S, h, \delta}$ for $h$, together with respective functions $\psi_{t} \in C(D)$ with $\rho\left(h, \psi_{t}\right)<\delta$ such that $\chi(x)_{t+1}=\psi_{t}\left(\chi(x)_{t}\right)$ for all $x \in D$ and all integer $t \geq 0$. Note that then $\rho\left(\psi_{t}, g\right)<\eta$.

Fix any $\delta$-pseudo-orbit $\left\{x_{t}\right\}_{t=0}^{\infty}$ of $h$. Directly from Lemma 15 we obtain that for all $t \geq 0$, if $x_{t} \in U_{i}$ then $x_{t+1} \in \bigcup_{j=1}^{k(i)} V_{i, j}$. Moreover, using arguments similar to those used in the proof of Theorem 10, we select sequences $\{\eta(t)\}_{t=0}^{\infty} \subset\{1, \ldots, n\}$ and $\{\zeta(t)\}_{t=0}^{\infty} \subset \mathbb{N}$ such that for every $t \geq 0$ the following conditions are satisfied:

$$
x_{t} \in U_{\eta(t)}, \quad x_{t+1} \in U_{\eta(t+1)} \in\left\{V_{\eta(t), j}\right\}_{j=1}^{k(\eta(t)},
$$

and

$$
S_{\eta(t+1), \xi(t+1)} \subset h\left(S_{\eta(t), \xi(t)}\right), \quad S_{\eta(t+1), \xi(t+1)} \subset \psi_{t}\left(S_{\eta(t), \xi(t)}\right) .
$$

By the above, there exist points

$$
y \in \bigcap_{t=0}^{\infty} h^{-t}\left(S_{\eta(t), \xi(t)}\right), \quad z \in S_{\eta(0), \xi(0)} \cap \bigcap_{t=1}^{\infty}\left(\psi_{t-1} \circ \cdots \circ \psi_{0}\right)^{-1}\left(S_{\eta(t), \xi(t)}\right),
$$


which implies that for each $t \geq 0$ we have $h^{t}(y), \chi(z)_{t} \in S_{\eta(t), \xi(t)} \subset U_{\eta(t)}$. In particular,

$$
d\left(h^{t}(y), x_{t}\right) \leq \operatorname{diam}\left(U_{\eta(t)}\right)<\varepsilon \text { and } d\left(\chi(z)_{t}, x_{t}\right) \leq \operatorname{diam}\left(U_{\eta(t)}\right)<\varepsilon
$$

for every integer $t \geq 0$, which ends the proof.

Theorem 19 For any dendrite $D$, the set of continuous maps with the $\mathscr{T}_{S}$-bi-shadowing property is residual in $C(D)$.

Proof The proof generally follows the same steps as the proof of Theorem 11, with the main difference that instead of Theorem 10 we apply Theorem 18 . We leave the details to the reader.

\subsection{S-Limit Shadowing is $\mathscr{C}^{0}$-Dense on Dendrites}

We finish this section by proving the results regarding density of $s$-limit shadowing for continuous maps on dendrites. We start with the following auxiliary lemma.

Lemma 20 Let $D$ be a dendrite, $f \in C(D)$ and $\gamma<\varepsilon / 8$ be a positive constant such that if $d(x, y)<\gamma$ then $d(f(x), f(y))<\varepsilon / 8$. Let $T \subset D$ be a tree such that $\rho(R, i d)<\gamma$, where $R$ denotes the first point map for T. Let $\left\{\tilde{U}_{i}\right\}_{i=1}^{n}$ be a cover of $T$ meeting the assumptions of Lemma 7 for the map $\left.R \circ f\right|_{T} \in C(T)$ and the constant $\varepsilon / 4$. Put $U_{i}=R^{-1}\left(\tilde{U}_{i}\right)$ and take a respective family of sets $\left\{S_{i, j}\right\}$ for $i \in\{1, \ldots, n\}$, $j \in\{1, \ldots, k(i)\}$, provided by Lemma 7 as well as a map $g \in C(D)$ provided by Lemma 15 for the above setting.

Then for any tree $Y$ such that $T \subset Y \subset D$ and any $\eta>0$ there exists a cover $\left\{W_{s}\right\}_{s=1}^{m}$ of $Y$ consisting of pairwise distinct arcs satisfying (3.2), such that

$\max \left\{\operatorname{diam}\left(W_{s}\right), \operatorname{diam}\left(g\left(W_{s}\right)\right)\right\}<\min \{\eta, \Gamma(Y) / 3\}$ for each $s \in\{1, \ldots, m\}$,

and the following conditions hold:

(1) for each $s \in\{1, \ldots, m\}$ there is $i \in\{1, \ldots, n\}$ such that

$$
R\left(W_{s}\right) \subset \tilde{U}_{i},
$$

(2) for each $i \in\{1, \ldots, n\}$ we have

$$
\bigcup\left\{W \in\left\{W_{s}\right\}_{s=1}^{m} \mid W \subset U_{i}\right\}=U_{i},
$$

(3) for each $i \in\{1, \ldots, n\}$ and each $j \in\{1, \ldots, k(i)\}$ we have

$$
\bigcup\left\{W \in\left\{W_{s}\right\}_{s=1}^{m} \mid W \subset S_{i, j}\right\}=S_{i, j} .
$$

Proof The proof is left to the reader as an easy exercise. 
Remark 21 Under the assumptions of Lemma 20, if $Q: D \rightarrow Y$ is the first point map then $R=\left.R\right|_{Y} \circ Q=R \circ Q$. In particular, the family $\left\{Q^{-1}\left(W_{s}\right)\right\}$ is a cover of $D$ that refines $\left\{U_{i}\right\}$.

Now, we are ready to prove our last result for continuous maps on dendrites.

Theorem 22 For any dendrite D, the set of continuous maps with the s-limit shadowing property is $\mathscr{C}^{0}$-dense in $C(D)$.

Proof The proof is analogous to the proof of Theorem 13. Specifically, we apply Lemma 15 for some cover $\left\{\tilde{U}_{i}^{1}\right\}$ of appropriately chosen tree $T^{1} \subset D$ and so we obtain a cover $\left\{U_{i}\right\}:=\left\{U_{i}^{1}\right\}$ of $D$, consisting of sets with sufficiently small diameters. Then we take a larger tree $T^{2} \supset T^{1}$ provided by Lemma 14, together with its suitable cover $\left\{\tilde{U}_{i}^{2}\right\}$ that allows us to apply Lemma 20 and Remark 21 in order to construct an appropriate refinement $\left\{U_{i}^{2}\right\}$ of the cover $\left\{U_{i}^{1}\right\}$. This way we proceed inductively, adjusting steps from the proof of Theorem 13 accordingly.

\section{Shadowing is $\mathscr{C}^{0}$-Generic on Chainable Continua Retractable to Arcs}

Let $(X, d)$ be a continuum. A chain in $X$ is a finite, non-empty, indexed collection $\mathscr{C}=\left\{U_{1}, \ldots, U_{n}\right\}$ of open subsets of $X$ such that $U_{i} \cap U_{j} \neq \varnothing$ if and only if $|i-j| \leq 1$. If, additionally, $\operatorname{diam}\left(U_{i}\right)<\varepsilon$ for some $\varepsilon>0$ and every $i \in\{1, \ldots, n\}$, then we say that $\mathscr{C}$ is an $\varepsilon$-chain in $X$. A chain $\mathscr{C}$ (or an $\varepsilon$-chain $\mathscr{C}$, respectively) which is also a cover of $X$ is called a chain cover of $X$ (or an $\varepsilon$-chain cover of $X$, respectively).

We say that $X$ is a chainable continuum, if for every $\varepsilon>0$ there exists an $\varepsilon$-chain cover of $X$. It is well known (see, e.g., [20, Lemma 12.10]) that if a continuum $X$ is chainable, then for every $\varepsilon>0$ there is an $\varepsilon$-chain cover $\mathscr{C}=\left\{U_{1}, \ldots, U_{n}\right\}$ of $X$ such that $\overline{U_{i}} \cap \overline{U_{j}}=\emptyset$, provided that $|i-j| \geq 2$. Additionally, without loss of generality we may also assume that $\mathscr{C} \backslash\left\{U_{i}\right\}$ is not a cover of $X$ for every $i \in\{1, \ldots, n\}$, which is equivalent to saying that $U_{1} \backslash U_{2} \neq \emptyset$ and $U_{n} \backslash U_{n-1} \neq \emptyset$. Any cover $\mathscr{C}$ satisfying the above two properties is called a taut cover of $X$. It is also not hard to verify that if $\left\{U_{1}, \ldots, U_{n}\right\}$ is a chain cover of $X$ and $C \subset X$ is a continuum such that $C \cap U_{s} \neq \varnothing$ and $C \cap U_{t} \neq \emptyset$ for some $s<t$, then $C \cap U_{l} \neq \emptyset$ for every $s \leq l \leq t$. The reader not familiar with the continuum theory is referred to the monograph [20].

The aim of this section is to prove the following theorem, which is one of the main results of this paper.

Theorem 23 Let $X$ be a chainable continuum and assume that for every $\gamma>0$ there are an arc $Y \subset X$ and a retraction $R: X \rightarrow Y$, such that $\rho(R, i d)<\gamma$. Then the set of continuous maps with the $\mathscr{T}_{S}$-bi-shadowing property is residual in $C(X)$.

Proof Fix $f \in C(X)$ and $\varepsilon>0$. Let $\eta<\varepsilon / 9$ be a positive constant such that if $d(x, y)<3 \eta$ then $d(f(x), f(y))<\varepsilon / 9$. Let $\mathscr{C}=\left\{U_{1}, \ldots, U_{n}\right\}$ be a taut $\eta$-chain cover of $X$. Note that then

$$
\operatorname{diam}\left(f\left(U_{i-1} \cup U_{i} \cup U_{i+1}\right)\right)<\varepsilon / 9 \text { for } i \in\{2, \ldots, n-1\} \text {. }
$$


Take a positive constant $\alpha$ such that $\alpha<\min \left\{\operatorname{dist}\left(\overline{U_{i}}, \overline{U_{j}}\right):|i-j| \geq 2\right\}$ and let $\beta>0$ be a Lebesgue number of the $\operatorname{cover} \mathscr{C}$. Fix any $\gamma>0$ such that

$$
\gamma<\min \{\alpha / 3, \beta / 3\}<\beta \leq \max \left\{\operatorname{diam}\left(U_{i}\right)\right\}<\varepsilon / 9 .
$$

By the assumptions there are an $\operatorname{arc} \subset X$ and a retraction $R: X \rightarrow Y$ with $\rho(R, i d)<\gamma$. Furthermore, if $a, b \in X$ with $d(a, b)>\alpha$ then $d(R(a), R(b))>$ $\alpha-2 \gamma>0$, which shows that $\overline{R\left(U_{i}\right)} \cap \overline{R\left(U_{j}\right)}=\emptyset$ if and only if $|i-j| \geq 2$.

Take any taut $\gamma$-chain cover $\mathscr{C}^{*}=\left\{W_{1}^{*}, \ldots, W_{k}^{*}\right\}$ of $Y$ consisting of connected sets (it, consequently, means that each set $\overline{W_{j}^{*}}$ is an arc). Then there exists $\omega>0$ such that for every $i \in\{1, \ldots, k\}$ there is a point $q_{i} \in Y$ satisfying conditions

$$
B_{\omega}\left(q_{i}\right) \cap Y \subset W_{i}^{*} \backslash \bigcup_{j \neq i} \overline{W_{j}^{*}}
$$

and

$$
\omega<\min \left\{\operatorname{dist}\left(\overline{W_{i}^{*}}, \overline{W_{j}^{*}}\right):|i-j| \geq 2\right\} .
$$

Put $W_{i}=R^{-1}\left(W_{i}^{*}\right)$ for all $i \in\{1, \ldots, k\}$ and observe that $W_{i}^{*}=R\left(W_{i}\right)=W_{i} \cap Y$. Then, by (4.1), $\mathscr{D}=\left\{W_{1}, \ldots, W_{k}\right\}$ is $3 \gamma$-chain cover of $X$ and, additionally, $\mathscr{D}$ refines $\mathscr{C}$ because $3 \gamma<\beta$. In particular, for each $i \in\{1, \ldots, k\}$ there is $s(i)$ such that $W_{i} \subset U_{s(i)}$.

Define $F=R \circ f \circ R: X \rightarrow Y$ and for every $i \in\{1, \ldots, k\}$ put

$$
r(i)=\min \left\{j: F\left(\overline{W_{i}}\right) \cap W_{j}^{*} \neq \emptyset\right\}, \quad t(i)=\max \left\{j: F\left(\overline{W_{i}}\right) \cap W_{j}^{*} \neq \emptyset\right\} .
$$

Then, obviously, $r(i) \leq t(i)$ and

$$
F\left(\overline{W_{i}}\right) \subset W_{r(i)}^{*} \cup W_{r(i)+1}^{*} \cup \ldots \cup W_{t(i)}^{*} \subset W_{r(i)} \cup W_{r(i)+1} \cup \ldots \cup W_{t(i)}
$$

Moreover, since $F\left(\overline{W_{i}}\right)=R\left(f\left(\overline{W_{i}^{*}}\right)\right)$ is a continuum, $F\left(\overline{W_{i}}\right) \cap W_{j}^{*} \neq \varnothing$ for all $r(i) \leq j \leq t(i)$. Hence, by (5.1), for any $i \in\{1, \ldots, k\}$ we have

$$
\begin{aligned}
\operatorname{diam}\left(F\left(\overline{W_{i}}\right)\right) & \leq \operatorname{diam}\left(R\left(f\left(B_{\alpha / 3}\left(\overline{U_{s(i)}}\right)\right)\right)\right) \leq \operatorname{diam}\left(R\left(f\left(\bigcup_{|j-s(i)| \leq 1} U_{j}\right)\right)\right) \\
& \leq \varepsilon / 9+2 \gamma,
\end{aligned}
$$

which implies that

$$
\begin{aligned}
\operatorname{diam}\left(W_{r(i)} \cup \ldots \cup W_{t(i)}\right) & \leq \operatorname{diam}\left(F\left(\overline{W_{i}}\right)\right)+2 \max _{j} \operatorname{diam}\left(W_{j}\right) \\
& \leq \varepsilon / 9+2 \gamma+2 \varepsilon / 9 \leq \varepsilon / 3+2 \gamma .
\end{aligned}
$$

Take a positive constant $\mu<\min \left\{\delta_{W^{*}}, \omega / 7\right\}$, where $\delta_{W^{*}}$ is a Lebesgue number of the cover $\mathscr{C}^{*}$. Take any taut $\mu$-chain cover $\left\{V_{1}^{*}, \ldots, V_{m}^{*}\right\}$ of $Y$ consisting of connected 
sets (it, consequently, means that each set $\overline{V_{j}^{*}}$ is an arc). By (5.3), for every $i \in$ $\{1, \ldots, k\}$ there is $p(i)$ such that $q_{i} \in V_{p(i)}^{*}$. Reversing indexing of sets $V_{i}$, if required, we may assume that $p(1)<p(k)$. Now, if we put $j(1)=p(1)$ and $j(k)=p(k)-4$, then for $i=1$ and $i=k$ the following condition is satisfied:

$$
V_{j(i)+l}^{*} \subset B_{\omega}\left(q_{i}\right) \subset W_{i}^{*} \backslash \bigcup_{j \neq i} \overline{W_{j}^{*}}
$$

for $l \in\{0, \ldots, 4\}$. For $i \in\{2, \ldots, k-1\}$ take inductively as $j(i)$ the smallest integer $j(i)>j(i-1)$ such that $V_{j(i)+5}^{*} \cap \overline{W_{i+1}^{*}} \neq \emptyset$. Since $V_{j(i-1)}^{*} \subset W_{i-1}^{*}$ we see that $V_{j(i)+l}^{*} \subset \bigcup_{j \leq i} W_{j}^{*}$ for $l \in\{0, \ldots, 4\}$, which obviously implies that (5.7) holds for that particular $i$. It is also clear that each $j(i)$ constructed this way satisfies condition $j(i)<j(k)$ and therefore

$$
i_{1}<i_{2} \Longrightarrow j\left(i_{1}\right)<j\left(i_{2}\right) \text {. }
$$

Define $V_{i}=R^{-1}\left(V_{i}^{*}\right)$ for all $i \in\{1, \ldots, k\}$ and observe that $V_{i}^{*}=R\left(V_{i}\right)=V_{i} \cap Y$. Directly by definition, $\left\{V_{1}, \ldots, V_{m}\right\}$ is a chain cover of $X$ that refines $\left\{W_{1}, \ldots, W_{k}\right\}$ and for all $i \in\{1, \ldots, k\}$ we have

$$
V_{j(i)+l} \subset W_{i} \backslash \bigcup_{j \neq i} \overline{W_{j}} .
$$

Since the closure of the set $W_{r(i)}^{*} \cup W_{r(i)+1}^{*} \cup \ldots \cup W_{t(i)}^{*}$ is an arc, we easily construct (by "stretching" the arcs $\overline{V_{j(i)}^{*}}, \overline{V_{j(i)+2}^{*}}$ and $\overline{V_{j(i)+4}^{*}}$ inside $W_{r(i)}^{*} \cup W_{r(i)+1}^{*} \cup \ldots \cup W_{t(i)}^{*}$ ) a continuous map $G: Y \rightarrow Y$ such that for all $i \in\{1, \ldots k\}$ we have

$$
\begin{aligned}
G(x)= & F(x) \text { for all } x \in Y \backslash \bigcup_{i} \bigcup_{l=0}^{4} \overline{V_{j(i)+l}^{*}}, \\
& G\left(\overline{W_{i}^{*}}\right) \subset W_{r(i)}^{*} \cup W_{r(i)+1}^{*} \cup \ldots \cup W_{t(i)}^{*} \subset W_{r(i)} \cup W_{r(i)+1} \cup \ldots \cup W_{t(i)}
\end{aligned}
$$

and

$$
G\left(\overline{V_{j(i)+1}^{*}}\right) \subset V_{j(r(i))}^{*} \subset V_{j(r(i))}, \quad G\left(\overline{V_{j(i)+3}^{*}}\right) \subset V_{j(t(i))+4}^{*} \subset V_{j(t(i))+4}
$$

Put $g=G \circ R$ and observe that, by (5.10), we obtain

$$
g\left(\overline{V_{j(i)+1}}\right) \subset W_{r(i)} \text { and } g\left(\overline{V_{j(i)+3}}\right) \subset W_{t(i)}
$$

Now note that for any $x \in X$ there is $i \in\{1, \ldots, k\}$ such that $x \in W_{i}$ and so (5.5), (5.6) and (5.9) give the following sequence of estimates:

$$
d(F(x), G \circ R(x)) \leq \operatorname{diam}\left(W_{r(i)} \cup \ldots \cup W_{t(i)}\right) \leq \varepsilon / 3+2 \gamma .
$$


Similarly, by the definition of $\gamma$ and $R$ we obtain

$$
d(f(x), F(x)) \leq d\left(f(x), f(R(x))+d\left(f(R(x)), R(f(R(x))) \leq \frac{\varepsilon}{9}+\gamma .\right.\right.
$$

Summing up the above, we conclude that

$$
\rho(f, g) \leq \rho(f, F)+\rho(F, g) \leq \frac{\varepsilon}{9}+\gamma+\frac{\varepsilon}{3}+2 \gamma<\varepsilon .
$$

Clearly, by (5.9) and (5.10) there is a constant $\delta>0$ such that

$$
B_{3 \delta}\left(g\left(\overline{V_{j(i)+1}}\right)\right) \subset V_{j(r(i))}, \quad B_{3 \delta}\left(g\left(\overline{V_{j(i)+3}}\right)\right) \subset V_{j(t(i))+4}
$$

and

$$
B_{3 \delta}\left(g\left(\overline{W_{i}}\right)\right) \subset \bigcup_{j=r(i)}^{t(i)} W_{j} .
$$

Assume additionally that $2 \delta$ is a Lebesgue number for the cover $\left\{W_{1}, \ldots, W_{k}\right\}$.

Let $h \in C(X)$ be a map satisfying $\rho(h, g)<\delta$ and let $\left\{x_{s}\right\}_{s=0}^{\infty}$ be a $\delta$-pseudoorbit of $h$. Take any sequence of continuous maps $\left\{h_{s}\right\}_{s=1}^{\infty}$ with $\rho\left(h_{s}, h\right)<\delta$ and note that, by (5.12) and the fact that $2 \delta$ is a Lebesgue number of $\left\{W_{1}, \ldots, W_{k}\right\}$ there exists a sequence of indices $\{n(s)\}_{s=0}^{\infty}$ such that for every $s$ we have $x_{s} \in W_{n(s)}$, $h\left(x_{s}\right) \in W_{n(s+1)}, h_{s}\left(x_{s}\right) \in W_{n(s+1)}$ and $r(n(s)) \leq n(s+1) \leq t(n(s))$.

Let $I_{0}$ be any arc in $Y$ with endpoints in $V_{j(n(0))+1}^{*}$ and $V_{j(n(0))+3}^{*}$. Obviously, $I_{0} \subset V_{j(n(0))+1}^{*} \cup V_{j(n(0))+2}^{*} \cup V_{j(n(0))+3}^{*} \subset W_{n(0)}$, because each set $\overline{V_{i}^{*}}$ is an arc.

Now suppose that for some $s \geq 0$ we have already constructed an arc $I_{s} \subset Y$ such that

$$
\left(h_{s} \circ \cdots \circ h_{0}\right)\left(I_{S}\right) \cap V_{j(n(s))+1} \neq \emptyset, \quad\left(h_{s} \circ \cdots \circ h_{0}\right)\left(I_{s}\right) \cap V_{j(n(s))+3} \neq \varnothing
$$

and

$$
\left(h_{s} \circ \cdots \circ h_{0}\right)\left(I_{s}\right) \subset V_{j(n(s))+1} \cup V_{j(n(s))+2} \cup V_{j(n(s))+3} \subset W_{n(s)},
$$

where for technical reasons we put here $h_{0}=\mathrm{id}$. Then $\left(h_{s+1} \circ h_{s} \circ \cdots \circ h_{1}\right)\left(I_{s}\right) \cap$ $h_{s+1}\left(V_{j(n(s))+1}\right) \neq \emptyset$ and, using (5.11), we obtain

$$
\left(h_{s+1} \circ \cdots \circ h_{1}\right)\left(I_{S}\right) \cap V_{j(r(n(s)))} \neq \emptyset .
$$

In the same way we obtain

$$
\left(h_{s+1} \circ \cdots \circ h_{1}\right)\left(I_{S}\right) \cap V_{j(t(n(s)))+4} \neq \emptyset .
$$

But the sets $V_{i}$ form a chain cover of $X$ and the set $\left(h_{s+1} \circ \cdots \circ h_{1}\right)\left(I_{s}\right)$ is a continuum, so, using (5.8), we obtain 


$$
\begin{aligned}
& \left(h_{s+1} \circ \cdots \circ h_{1}\right)\left(I_{s}\right) \cap V_{j(n(s+1))+1} \neq \varnothing \text { and } \\
& \left(h_{s+1} \circ \cdots \circ h_{1}\right)\left(I_{s}\right) \cap V_{j(n(s+1))+3} \neq \emptyset .
\end{aligned}
$$

Fix any two points $a, b \in I_{S}$ such that

$$
\left(h_{s+1} \circ \cdots \circ h_{1}\right)(a) \in V_{j(n(s+1))+1} \quad \text { and } \quad\left(h_{s+1} \circ \cdots \circ h_{1}\right)(b) \in V_{j(n(s+1))+3} .
$$

Take a subarc of $I_{s}$ with the endpoints $a$ and $b$, together with its parametrization $\tau:[0,1] \rightarrow Y$ satisfying $\tau(0)=a$ and $\tau(1)=b$. Put

$$
\begin{aligned}
& t_{1}=\sup \left\{t \in(0,1):\left(h_{s+1} \circ \cdots \circ h_{1}\right)(\tau(t)) \in V_{j(n(s+1))+1}\right\}, \\
& t_{2}=\inf \left\{t \in\left(t_{1}, 1\right):\left(h_{s+1} \circ \cdots \circ h_{1}\right)(\tau(t)) \in V_{j(n(s+1))+3}\right\} .
\end{aligned}
$$

Clearly,

$$
\left(h_{s+1} \circ \cdots \circ h_{1}\right)\left(\tau\left(t_{1}\right)\right) \in \overline{V_{j(n(s+1))+1}}, \quad\left(h_{s+1} \circ \cdots \circ h_{1}\right)\left(\tau\left(t_{2}\right)\right) \in \overline{V_{j(n(s+1))+3}},
$$

and so we can find sufficiently small $\zeta>0$ such that the arc $I_{s+1}=\tau\left(\left[t_{1}-\zeta, t_{2}+\zeta\right]\right) \subset$ $I_{S}$ satisfies

$$
\begin{aligned}
& \left(h_{s+1} \circ \cdots \circ h_{0}\right)\left(I_{s+1}\right) \cap V_{j(n(s+1))+1} \neq \varnothing, \\
& \left(h_{s+1} \circ \cdots \circ h_{0}\right)\left(I_{s+1}\right) \cap V_{j(n(s+1))+3} \neq \varnothing
\end{aligned}
$$

and

$$
\left(h_{s+1} \circ \cdots \circ h_{0}\right)\left(I_{s+1}\right) \subset V_{j(n(s+1))+1} \cup V_{j(n(s+1))+2} \cup V_{j(n(s+1))+3} \subset W_{n(s+1)} .
$$

Using the induction, we obtain a nested sequence of arcs

$$
I_{0} \supset I_{1} \supset I_{2} \supset \ldots,
$$

such that $\left(h_{s} \circ \cdots \circ h_{0}\right)\left(I_{s}\right) \subset W_{n(s)}$ for all $s \geq 0$. Hence there exists $z \in \bigcap_{s=0}^{\infty} I_{s}$, which by the construction satisfies

$$
d\left(\left(h_{s} \circ \cdots \circ h_{0}\right)(z), x_{s}\right) \leq \operatorname{diam}\left(W_{n(s)}\right)<\varepsilon / 9<\varepsilon .
$$

Take any $\delta$-method $\chi \in \mathscr{T}_{S, h, \delta}$ for $h$, together with respective functions $\psi_{t} \in C(X)$ such that $\rho\left(h, \psi_{t}\right)<\delta$ and $\chi(x)_{t+1}=\psi_{t}\left(\chi(x)_{t}\right)$ for each $x \in X$ and all integers $t \geq 0$. Additionally, for technical reasons, put $\psi_{-1}=$ id. Following the preceding arguments, it is not hard to show that the $\delta$-pseudo-orbit $\left\{x_{s}\right\}$ is $\varepsilon$-traced by some orbit of $h$ as well as by some $\delta$-pseudo-orbit of $h$ that is produced by $\chi$. Indeed, it is enough to apply the above reasoning for the following sequences of continuous maps: $\{h\}_{s=1}^{\infty}$ and $\left\{\psi_{s-1}\right\}_{s=1}^{\infty}$, and then $\varepsilon$-tracing of $\left\{x_{s}\right\}$ is guaranteed in the first case by the orbit $\left\{h^{s}(z)\right\}_{s=0}^{\infty}$ and in the second case by the $\delta$-pseudo-orbit $\chi(z)=$ $\left\{\left(\psi_{s-1} \circ \cdots \circ \psi_{-1}\right)(z)\right\}_{s=0}^{\infty}$. Hence we conclude that for any $n \in \mathbb{N}$ taking the set $A_{n}$ 


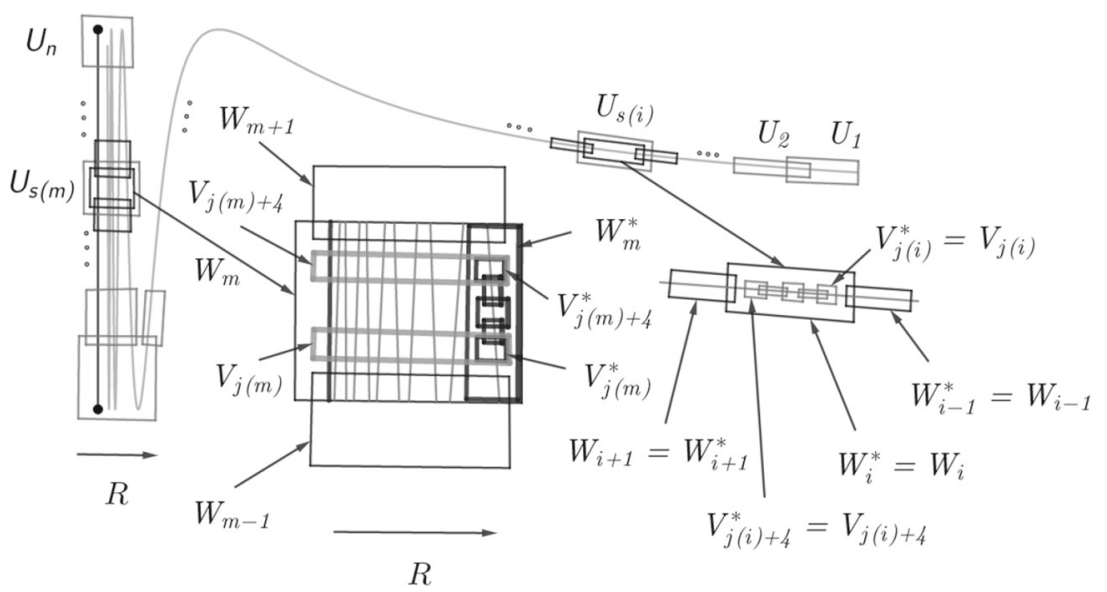

Fig. 5 The idea of a construction of chain covers from the proof of Theorem 23

consisting of continuous maps $f$ for which there is $\delta>0$ such that, having established a $\delta$-method $\chi \in \mathscr{T}_{S, f, \delta}$ for $f$, any $\delta$-pseudo-orbit of $f$ is $1 / n$-traced by some orbit of $f$ as well as by some $\delta$-pseudo-orbit of $f$ that is produced by $\chi$, we obtain that $A_{n}$ contains a subset that is open and dense in $C(X)$. But then the set $A=\bigcap_{n=1}^{\infty} A_{n}$ is residual in $C(X)$, while all its elements have the $\mathscr{T}_{S}$-bi-shadowing property. This completes the proof.

Remark 24 An example of a continuum satisfying the assumptions of Theorem 23 is the closure of $\sin (1 / x)$-curve on any interval $(0, a]$, where $a>0$. In this case, the concept of a construction of consecutive taut chain covers (as in the above proof) is presented in Fig. 5.

Remark 25 In [7] it was proved that many tent maps have the shadowing property. The inverse limit of each of these maps, restricted to the core, defines a Knaster continuum (the Knaster bucket handle continuum is obtained using then map with slope 2), and it is not hard to see that each of these continua satisfies assumptions of Theorem 23. On the other hand, shift homeomorphism defined by associated tent map has the shadowing property if and only if the tent map has it, too. Therefore, some of these homeomorphism have, and some other does not have, the shadowing property. But by Theorem 23 on each of these continua shadowing is generic, which, in particular, means that close to any shift homeomorphism there is a continuous map with the shadowing property.

Acknowledgements P. Oprocha was partially supported by the Faculty of Applied Mathematics AGH UST statutory tasks within subsidy of Ministry of Science and Higher Education, Project No. 11.11.420.004, by the Ministry of Education, Youth and Sports from the National Programme of Sustainability (NPU II) Project "IT4Innovations excellence in science-LQ1602" and Project 1RP201824 "Complex topological structures." All the pictures in the paper were made by the authors with the help of GeoGebra software, available at https://www.geogebra.org/, which we gratefully acknowledge. After the paper was accepted, we noticed a recent preprint by Meddaugh [17], who proves that shadowing is generic for graphites (roughly speaking, locally connected spaces which can be retracted to subgraphs by maps with small fibers). Clearly, classes of spaces considered here and in [17] have non-empty intersections and complements. 
Open Access This article is licensed under a Creative Commons Attribution 4.0 International License, which permits use, sharing, adaptation, distribution and reproduction in any medium or format, as long as you give appropriate credit to the original author(s) and the source, provide a link to the Creative Commons licence, and indicate if changes were made. The images or other third party material in this article are included in the article's Creative Commons licence, unless indicated otherwise in a credit line to the material. If material is not included in the article's Creative Commons licence and your intended use is not permitted by statutory regulation or exceeds the permitted use, you will need to obtain permission directly from the copyright holder. To view a copy of this licence, visit http://creativecommons.org/licenses/by/4.0/.

\section{References}

1. Akin, E., Hurley, M., Kennedy, J.A.: Dynamics of topologically generic homeomorphisms. Mem. Am. Math. Soc. 164, 130 (2003)

2. Anosov, D.V.: Geodesic flows on closed Riemann manifolds with negative curvature. In: Proceedings of the Steklov Institute of Mathematics, No. 90 (1967), translated from the Russian by S. Feder American Mathematical Society, Providence, R.I. (1969)

3. Arévalo, D., Charatonik, W.J., Pellicer Covarrubias, P., Simón, L.: Dendrites with a closed set of end points. Topol. Appl. 115, 1-17 (2001)

4. Bernardes, C.N., Darji, B.D.: Graph theoretic structure of maps of the Cantor space. Adv. Math. 231, 1655-1680 (2012)

5. Bowen, R.: $\omega$-limit sets for axiom a diffeomorphisms. J. Differ. Equ. 18, 333-339 (1975)

6. Brian, W., Meddaugh, J., Raines, B.E.: Shadowing is generic on dendrites, to appear in Discrete Contin. Dyn. Syst. Ser. S

7. Coven, E.M., Kan, I., Yorke, J.A.: Pseudo-orbit shadowing in the family of tent maps. Trans. Am. Math. Soc. 308, 227-241 (1988)

8. Denker, M., Grillenberger, Ch., Sigmund, K.: Ergodic theory on compact spaces. Lectures Notes in Math., vol. 527. Springer, Berlin (1976)

9. Kato, H.: The nonexistence of expansive homeomorphisms of chainable continua. Fund. Math. 149, 119-126 (1996)

10. Kennedy, J., Yorke, J.A.: Basins of Wada, Nonlinear science: the next decade (Los Alamos, NM, 1990). Physica D 51, 213-225 (1991)

11. Kościelniak, P.: On genericity of shadowing and periodic shadowing property. J. Math. Anal. Appl. 310, 188-196 (2005)

12. Kościelniak, P.: On the genericity of chaos. Topol. Appl. 154, 1951-1955 (2007)

13. Kościelniak, P., Mazur, M.: Genericity of inverse shadowing property. J. Differ. Equ. Appl. 16, 667-674 (2010)

14. Kościelniak, P., Mazur, M., Oprocha, P., Pilarczyk, P.: Shadowing is generic-a continuous map case. Discret. Contin. Dyn. Syst. 34, 3591-3609 (2014)

15. Krupski, P., Omiljanowski, K., Ungeheuer, K.: Chain recurrent sets of generic mappings on compact spaces. Topol. Appl. 202, 251-268 (2016)

16. Mazur, M., Oprocha, P.: $S$-limit shadowing is $\mathscr{C}^{0}$-dense. J. Math. Anal. Appl. 408, 465-475 (2013)

17. Meddaugh, J.: On genericity of shadowing in one-dimensional continua (2018). preprint arXiv: $1810.02262 \mathrm{v} 1$

18. Mizera, I.: Generic properties of one-dimensional dynamical systems. Ergodic theory and related topics, III (Güstrow, 1990), Lecture Notes in Math. 1514. Springer, Berlin, pp. 163-173 (1992)

19. Moise, E.E.: Geometric Topology in Dimensions 2 and 3, Graduate Texts in Mathematics, vol. 47. Springer, New York (1977)

20. Nadler Jr., S.B.: Continuum Theory. An introduction, Monographs and Textbooks in Pure and Applied Mathematics, vol. 158. Marcel Dekker, New York (1992)

21. Nikiel, J.: Topologies on pseudo-trees and applications. Mem. Am. Math. Soc. 82, 116 (1989)

22. Odani, K.: Generic homeomorphisms have the pseudo-orbit tracing property. Proc. Am. Math. Soc. 110, 281-284 (1990)

23. Omiljanowski, K., Zafiridou, S.: Universal completely regular dendrites. Colloq. Math. 103, 149-154 (2005) 
24. Palmer, K.: Shadowing in Dynamical Dystems. Theory and Applications, Mathematics and its Applications, vol. 501. Kluwer Academic Publishers, Dordrecht (2000)

25. Pilyugin, SYu.: Inverse shadowing by continuous methods. Discret. Contin. Dyn. Syst. 8, 29-38 (2002)

26. Pilyugin, SYu.: Shadowing in Dynamical Systems. Lecture Notes in Math., vol. 1706. Springer, Berlin (1999)

27. Pilyugin, SYu.: The Space of Dynamical Systems with the $\mathscr{C}^{0}$-Topology. Lecture Notes in Math., vol. 1571. Springer, Berlin (1994)

28. Pilyugin, SYu., Plamenevskaya, O.B.: Shadowing is generic. Topol. Appl. 97, 253-266 (1999)

29. Pilyugin, SYu., Sakai, K.: Shadowing and Hyperbolicity. Lecture Notes in Math., vol. 2193. Springer, Cham (2017)

30. Pol, R.: The works of Stefan Mazurkiewicz in topology. Handbook of the history of general topology, Vol. 2 (San Antonio, TX, 1993), Hist. Topol. 2, Kluwer Acad. Publ., Dordrecht, pp. 415-430 (1998)

31. Sobolewski, M.: Means on chainable continua. Proc. Am. Math. Soc. 136, 3701-3707 (2008)

32. Yano, K.: Generic homeomorphisms of $S_{1}$ have the pseudo-orbit tracing property. J. Fac. Sci. Univ. Tokyo Sect. IA Math. 34, 51-55 (1987)

33. Zgliczyński, P.: Fixed point index for iterations of maps, topological horseshoe and chaos. Topol. Methods Nonlinear Anal. 8, 169-177 (1996)

34. Zgliczyński, P., Gidea, M.: Covering relations for multidimensional dynamical systems. J. Differ. Equ. 202, 32-58 (2004)

Publisher's Note Springer Nature remains neutral with regard to jurisdictional claims in published maps and institutional affiliations.

\section{Affiliations}

\section{Piotr Kościelniak $^{1} \cdot$ Marcin Mazur $^{1} \cdot$ Piotr Oprocha $^{2,3}$ (D) Łukasz Kubica $^{1}$}

Piotr Kościelniak

piotr.koscielniak@uj.edu.pl

Marcin Mazur

marcin.mazur@uj.edu.pl

Łukasz Kubica

lukasz2.kubica@student.uj.edu.pl

1 Department of Applied Mathematics, Institute of Mathematics, Faculty of Mathematics and Computer Science, Jagiellonian University, ul. Łojasiewicza 6, 30-348 Kraków, Poland

2 Faculty of Applied Mathematics, AGH University of Science and Technology, al. Mickiewicza 30, 30-059 Kraków, Poland

3 National Supercomputing Centre IT4Innovations, Division of the University of Ostrava, Institute for Research and Applications of Fuzzy Modeling, 30. Dubna 22, 70103 Ostrava, Czech Republic 